\title{
HIFLUGCS: X-ray luminosity-dynamical mass relation and its implications for mass calibrations with the SPIDERS and 4MOST surveys
}

\author{
Yu-Ying Zhang ${ }^{1, \dagger}$, Thomas H. Reiprich ${ }^{1}$, Peter Schneider ${ }^{1}$, Nicolas Clerc $^{2}$, Andrea Merloni ${ }^{2}$, Axel Schwope $^{3}$, \\ Katharina Borm ${ }^{1}$, Heinz Andernach ${ }^{4}$, César A. Caretta ${ }^{4,5}$, and Xiang-Ping $\mathrm{Wu}^{6}$ \\ 1 Argelander-Institut für Astronomie, Universität Bonn, Auf dem Hügel 71, 53121 Bonn, Germany \\ e-mail: reiprich@astro.uni-bonn.de \\ 2 Max-Planck-Institut für extraterrestrische Physik, Giessenbachstraße, 85748 Garching, Germany \\ 3 Leibniz-Institut für Astrophysik Potsdam (AIP), An der Sternwarte 16, 14482 Potsdam, Germany \\ ${ }^{4}$ Departamento de Astronomía, Universidad de Guanajuato, AP 144, Guanajuato CP 36000, Mexico \\ 5 Laboratoire d'Astrophysique de Marseille, Aix-Marseille Université, 38 rue Frédéric Juliot-Curie, 13388 Marseille, France \\ ${ }^{6}$ National Astronomical Observatories, Chinese Academy of Sciences, 100012 Beijing, PR China \\ Received 19 May 2016 / Accepted 23 August 2016
}

\begin{abstract}
We present the relation of X-ray luminosity versus dynamical mass for 63 nearby clusters of galaxies in a flux-limited sample, the HIghest X-ray FLUx Galaxy Cluster Sample (HIFLUGCS, consisting of 64 clusters). The luminosity measurements are obtained based on 1.3 Ms of clean XMM-Newton data and ROSAT pointed observations. The masses are estimated using optical spectroscopic redshifts of 13647 cluster galaxies in total. We classify clusters into disturbed and undisturbed based on a combination of the X-ray luminosity concentration and the offset between the brightest cluster galaxy and X-ray flux-weighted center. Given sufficient numbers (i.e., $\geq 45$ ) of member galaxies when the dynamical masses are computed, the luminosity versus mass relations agree between the disturbed and undisturbed clusters. The cool-core clusters still dominate the scatter in the luminosity versus mass relation even when a core-corrected X-ray luminosity is used, which indicates that the scatter of this scaling relation mainly reflects the structure formation history of the clusters. As shown by the clusters with only few spectroscopically confirmed members, the dynamical masses can be underestimated and thus lead to a biased scaling relation. To investigate the potential of spectroscopic surveys to follow up highredshift galaxy clusters or groups observed in X-ray surveys for the identifications and mass calibrations, we carried out Monte Carlo resampling of the cluster galaxy redshifts and calibrated the uncertainties of the redshift and dynamical mass estimates when only reduced numbers of galaxy redshifts per cluster are available. The resampling considers the SPIDERS and 4MOST configurations, designed for the follow-up of the eROSITA clusters, and was carried out for each cluster in the sample at the actual cluster redshift as well as at the assigned input cluster redshifts of $0.2,0.4,0.6$, and 0.8 . To follow up very distant clusters or groups, we also carried out the mass calibration based on the resampling with only ten redshifts per cluster, and redshift calibration based on the resampling with only five and ten redshifts per cluster, respectively. Our results demonstrate the power of combining upcoming X-ray and optical spectroscopic surveys for mass calibration of clusters. The scatter in the dynamical mass estimates for the clusters with at least ten members is within $50 \%$.
\end{abstract}

Key words. cosmology: observations - methods: data analysis - galaxies: kinematics and dynamics - X-rays: galaxies: clusters galaxies: clusters: intracluster medium - surveys

\section{Introduction}

Galaxy clusters represent the place where astrophysics and cosmology meet: while their overall internal dynamics is dominated by gravity, the astrophysical processes taking place on galactic scales leave observable imprints on the diffuse hot gas trapped within their potential wells (Giacconi et al. 2009). Galaxy clusters (e.g., Vikhlinin et al. 2009a, b; Mantz et al. 2010), in combination with supernovae (e.g., Riess et al. 2011), cosmic microwave background (e.g., Bennett et al. 2013; Hinshaw et al. 2013; Planck Collaboration XVI 2016), baryon acoustic oscillations (e.g., Dawson et al. 2013) and cosmological weak lensing (e.g., Schrabback et al. 2010; Laureijs et al. 2011; Marian et al. 2011; Heymans et al. 2013) can constrain the dark energy equation-of-state parameter, $w$, at both late

$\dagger$ Deceased Dec. 11, 2016. We will miss her scientifically, her engagement in teaching, as well as her kindness. and early cosmological epochs. Upcoming experiments such as eROSITA will improve the statistical power of cluster cosmology by a few orders of magnitude (e.g., Predehl et al. 2010; Merloni et al. 2012). However, there are two major challenges in upcoming surveys for high-precision cluster cosmology: accurate mass calibrations and efficient follow-up, such as optical identifications and redshift measurements.

The cluster cosmological applications are degenerate with the mass calibrations of galaxy clusters (e.g., Zhang \& Wu 2003; Stanek et al. 2010; Merloni et al. 2012). Large X-ray surveys select galaxy clusters by their observables (e.g., Ebeling et al. 2000; Böhringer et al. 2004; Clerc et al. 2012; Hilton et al. 2012; Takey et al. 2013), particularly the X-ray luminosity $(L)$, and not by their masses $(M)$. The $L-M$ relation is required to recover the selection function in terms of cluster masses and predict the cluster masses, hence the cluster mass function. The relations of bolometric X-ray luminosity versus mass calibrated 
with different samples differ significantly (e.g., Chen et al. 2007; Maughan 2007; Pacaud et al. 2007; Pratt et al. 2009; Mantz et al. 2010; Reichert et al. 2011), in which the slope varies from $\sim 1.5$ to $\sim 2.0$. The predicted numbers of detected clusters in the eROSITA survey based on different calibrations of the $L-M$ scaling relations differ by up to a factor of two (e.g., Pillepich et al. 2012). In addition to the luminosity segregation between cool-core (CC) and non-cool-core (NCC) clusters, merging affects the properties of both the intracluster medium (ICM) and cluster galaxies (e.g., Poole et al. 2006; Evrard et al. 2008), but on different timescales (e.g., Roettiger et al. 1997). Mass estimates from optical spectroscopy and gravitational lensing, for instance, which are independent of the X-ray luminosity measurement, provide an X-ray blind reference of the cluster mass to calibrate the $L-M$ relation (e.g., Kellogg et al. 1990; Wu et al. 1998, 1999; Zhang et al. 2008; Leauthaud et al. 2010; Israel et al. 2014, 2015; von der Linden et al. 2014; Sereno 2015; Sereno \& Ettori 2015; Andreon et al. 2016). However, the dynamical mass estimates are sensitive to the cluster galaxy selection and member statistic and overestimate the mass of a merging cluster when the merger axis is along the line of sight (e.g., Biviano et al. 2006; Gifford \& Miller 2013; Old et al. 2013; Saro et al. 2013; Wu \& Huterer 2013; Rabitz et al. 2017).

The HIghest X-ray FLUx Galaxy Cluster Sample (HIFLUGCS, Reiprich \& Böhringer 2002) is a flux-limited sample of 64 clusters selected from the ROSAT All-Sky Survey (RASS; Ebeling et al. 2000; Böhringer et al. 2004). We analyze high-quality XMM-Newton and ROSAT pointed observations as well as optical spectroscopic redshifts of 13650 cluster galaxies for all 64 HIFLUGCS clusters. Excluding 2A 0335+096 with only three redshifts, we present the relation of X-ray luminosity versus dynamical mass for the 63 HIFLUGCS clusters and quantify the effect of mergers as well as CC systems on the $L-M$ relation. A simultaneous mass calibration and cosmological application procedure (e.g., Bocquet et al. 2015; Mantz et al. 2015) can break the degeneracy between them. This approach is promising for the use of the combined X-ray and optical surveys in the near future because a vast number of telescopes are dedicated to carry out the optical spectroscopic surveys of galaxy clusters, such as eBOSS/SPIDERS ${ }^{1}$ (Clerc et al. 2016) and $4 \mathrm{MOST}^{2}$. This method, however, relies not only on independent measurements of at least two cluster quantities, for example, X-ray luminosity and dynamical mass, but also on well-understood knowledge of the uncertainties and potential biases in the two measured quantities (e.g., Applegate et al. 2014; von der Linden et al. 2014; Planck Collaboration XIII 2016).

Furthermore, efficient follow-up identifications of a large number of galaxy clusters become one of the main tasks in upcoming surveys for high-precision cluster cosmology (e.g., Merloni et al. 2012; Nandra et al. 2013; Pointecouteau et al. 2013). To optimize the optical spectroscopy follow-up, it is invaluable to investigate in which redshift range existing and upcoming multiwavelength surveys are suitable to identify groups and clusters of galaxies and to measure their redshifts. For further applications of the optical spectroscopic follow-up, it is worth testing in which redshift range these multiwavelength surveys can provide accurate dynamical masses that are sufficient for the $L-M$ calibration.

In this paper, we simulate the optical spectroscopic follow-up of clusters by Monte Carlo (MC) resampling of the HIFLUGCS

\footnotetext{
1 wWW.sdss.org/surveys/eboss

2 wWW. 4 most.eu
}

cluster galaxy redshifts at hand according to eight optical spectroscopic setups. We calibrate the redshift and dynamical mass estimates and quantify in which redshift range the tested optical spectroscopic setups are reliable for measuring the cluster redshifts and dynamical masses. We organize the paper as follows. In Sect. 2 we briefly describe the sample, data, and analysis method. The results on the $L-M$ relation of the HIFLUGCS sample are presented in Sect. 3. The results on the redshift and dynamical mass calibrations using simulations of the re-sampled cluster galaxy redshifts are presented in Sect. 4. We summarize our conclusions in Sect. 5. Throughout the paper, we assume $\Omega_{\mathrm{m}}=0.3, \Omega_{\Lambda}=0.7, \sigma_{8}=0.81$, and $H_{0}=70 \mathrm{~km} \mathrm{~s}^{-1} \mathrm{Mpc}^{-1}$. We quote a $68 \%$ confidence level. Unless explicitly stated otherwise, we apply the BCES bisector regression method (Akritas \& Bershady 1996), taking into account measurement errors on both variables to determine the parameters and their errors in the fit.

\section{Sample, data, and analysis method}

The HIFLUGCS is a sample of galaxy clusters with an X-ray flux limit $(0.1-2.4 \mathrm{keV})$ of $2 \times 10^{-11} \mathrm{erg} / \mathrm{s} / \mathrm{cm}^{2}$ and Galactic latitude $|b| \geq 20.0$ degrees covering two-thirds of the sky (Reiprich \& Böhringer 2002; Hudson et al. 2010), selected from the ROSAT All-Sky Survey (RASS; Ebeling et al. 2000; Böhringer et al. 2004).

\subsection{X-ray data and analysis}

We analyzed nearly $4 \mathrm{Ms}$ of raw data from XMM-Newton for 63 clusters (Zhang et al. 2009, 2011, 2012). After cleaning and selecting the longest observation closest to the cluster center, we obtained $\sim 1.3 \mathrm{Ms}$ of XMM-Newton data for 59 clusters. We measured the X-ray properties for all 64 clusters in the HIFLUGCS by combining XMM-Newton and ROSAT data. The X-ray fluxweighted centroids are listed in Zhang et al. $(2011,2012)$ with the method described in Sect. 2.3 of Zhang et al. (2010).

\subsubsection{X-ray luminosity}

The X-ray luminosity was measured within the cluster radius, $r_{500}$. This radius is defined as that within which the matter overdensity is 500 times the critical density of the Universe. It was derived from the dynamical mass in Sect. 2.2.2 centered on the X-ray flux-weighted centroid except for 2A 0335+096, which has three galaxy redshifts in total and of which the cluster radius $r_{500}$ was derived from the scaling relation of mass versus gas mass. We note that Zhang et al. (2011) measured the $\mathrm{X}$-ray luminosity within the $r_{500}$ derived from the gas mass using the scaling relation of total mass versus gas mass derived in Pratt et al. (2009). To suppress the scatter of the $L-M$ relation caused by cool cores, we used the core-corrected X-ray luminosity ( $L^{\text {cor }}$ hereafter), which is derived from the integration of the surface brightness assuming a constant value of the surface brightness within $0.2 r_{500}$ equal to the value at $0.2 r_{500}$, $S_{\mathrm{X}}\left(R<0.2 r_{500}\right)=S_{\mathrm{X}}\left(0.2 r_{500}\right)$, following Zhang et al. (2007). Here $R$ is the projected cluster-centric distance. We note that this correction was only applied to determine the X-ray luminosity. We use the core-corrected bolometric X-ray luminosity (Table 1) for the relation of luminosity versus mass of the HIFLUGCS throughout the paper.

Some past work used a radius larger than $r_{500}$ (e.g., Reiprich \& Böhringer 2002) to derive the X-ray luminosity, and some used different methods to account for the cool cores (e.g., 
Y.-Y. Zhang et al.: HIFLUGCS: $L-M$ relation and its implications for mass calibrations with the SPIDERS and 4MOST surveys

Table 1. Cluster properties.

\begin{tabular}{|c|c|c|c|c|c|c|}
\hline Name & $n_{\text {gal }}$ & $\sigma\left(\mathrm{km} \mathrm{s}^{-1}\right)$ & $M_{500}\left(10^{14} M_{\odot}\right)$ & $L_{500}^{\text {cor }}\left(10^{44} \mathrm{erg} / \mathrm{s}\right)$ & $c_{\mathrm{L}}$ & $\Delta R / r_{500}$ \\
\hline A0085 & 350 & $963 \pm 39$ & $4.83 \pm 0.76$ & $7.60 \pm 0.43$ & 2.089 & 0.002 \\
\hline A0119 & 339 & $797 \pm 38$ & $2.67 \pm 0.47$ & $3.03 \pm 0.21$ & 5.383 & 0.014 \\
\hline A0133 & 137 & $725 \pm 44$ & $1.98 \pm 0.42$ & $1.45 \pm 0.09$ & 1.809 & 0.027 \\
\hline NGC 0507 & 110 & $503 \pm 33$ & $0.68 \pm 0.15$ & $0.11 \pm 0.01$ & 2.062 & 0.014 \\
\hline A0262 & 138 & $527 \pm 30$ & $0.78 \pm 0.16$ & $0.48 \pm 0.06$ & 2.705 & 0.006 \\
\hline A0400 & 114 & $647 \pm 40$ & $1.44 \pm 0.30$ & $0.39 \pm 0.03$ & 3.499 & 0.005 \\
\hline A0399 & 101 & $1223 \pm 75$ & $10.37 \pm 2.18$ & $5.63 \pm 0.48$ & 2.415 & 0.056 \\
\hline A0401 & 116 & $1144 \pm 74$ & $8.32 \pm 1.82$ & $11.76 \pm 0.91$ & 2.248 & 0.015 \\
\hline A3112 & 111 & $740 \pm 63$ & $2.09 \pm 0.57$ & $3.68 \pm 0.19$ & 1.569 & 0.024 \\
\hline NGC 1339/Fornax & 339 & $366 \pm 13$ & $0.27 \pm 0.04$ & $0.03 \pm 0.00$ & 2.595 & 0.001 \\
\hline 2A $0335+096$ & 3 & - & - & $1.43 \pm 0.12$ & 0.130 & 0.006 \\
\hline III Zw 054 & 45 & $657 \pm 62$ & $1.49 \pm 0.45$ & $0.43 \pm 0.06$ & 2.042 & 0.025 \\
\hline A3158 & 258 & $1044 \pm 45$ & $6.25 \pm 1.03$ & $5.41 \pm 0.41$ & 2.453 & 0.032 \\
\hline A0478 & 13 & $945 \pm 223$ & $4.45 \pm 3.20$ & $12.26 \pm 0.69$ & 1.572 & 0.003 \\
\hline NGC1550 & 22 & $263 \pm 34$ & $0.11 \pm 0.04$ & $0.12 \pm 0.02$ & 2.210 & 0.002 \\
\hline EXO 0422-086/RBS0540 & 42 & $298 \pm 59$ & $0.15 \pm 0.09$ & $0.89 \pm 0.04$ & 2.070 & 0.004 \\
\hline A3266 & 559 & $1174 \pm 41$ & $9.15 \pm 1.34$ & $8.33 \pm 0.34$ & 3.133 & 0.017 \\
\hline A0496 & 360 & $687 \pm 28$ & $1.71 \pm 0.27$ & $2.56 \pm 0.12$ & 2.001 & 0.004 \\
\hline A3376 & 165 & $798 \pm 46$ & $2.67 \pm 0.54$ & $1.28 \pm 0.09$ & 5.341 & 0.989 \\
\hline A3391 & 71 & $716 \pm 62$ & $1.92 \pm 0.54$ & $2.35 \pm 0.12$ & 3.801 & 0.043 \\
\hline A3395s & 215 & $841 \pm 39$ & $3.14 \pm 0.54$ & $1.74 \pm 0.13$ & 4.909 & 0.533 \\
\hline A0576 & 237 & $837 \pm 39$ & $3.12 \pm 0.54$ & $1.10 \pm 0.15$ & 2.056 & 0.040 \\
\hline A0754 & 470 & $928 \pm 34$ & $4.31 \pm 0.65$ & $6.30 \pm 0.31$ & 2.426 & 0.644 \\
\hline A0780/Hydra A & 37 & $687 \pm 82$ & $1.69 \pm 0.63$ & $2.94 \pm 0.14$ & 1.642 & 0.012 \\
\hline A1060 & 389 & $652 \pm 21$ & $1.48 \pm 0.21$ & $0.32 \pm 0.05$ & 1.932 & 0.001 \\
\hline A1367 & 343 & $639 \pm 24$ & $1.38 \pm 0.21$ & $1.01 \pm 0.05$ & 4.425 & 0.552 \\
\hline MKW4 & 145 & $417 \pm 37$ & $0.40 \pm 0.11$ & $0.18 \pm 0.02$ & 2.107 & 0.007 \\
\hline $\mathrm{ZwCl} 1215.1+0400$ & 154 & $889 \pm 51$ & $3.70 \pm 0.74$ & $5.61 \pm 0.26$ & 2.872 & 0.015 \\
\hline NGC 4636 & 115 & $224 \pm 12$ & $0.07 \pm 0.01$ & $0.00 \pm 0.00$ & 1.373 & 0.005 \\
\hline A3526/Centaurus & 235 & $486 \pm 24$ & $0.61 \pm 0.11$ & $0.68 \pm 0.09$ & 2.168 & 0.012 \\
\hline A1644 & 307 & $980 \pm 48$ & $5.13 \pm 0.92$ & $2.99 \pm 0.29$ & 3.841 & 0.021 \\
\hline A1650 & 220 & $794 \pm 43$ & $2.58 \pm 0.50$ & $5.30 \pm 0.21$ & 2.019 & 0.019 \\
\hline A1651 & 222 & $896 \pm 36$ & $3.77 \pm 0.60$ & $6.51 \pm 0.46$ & 1.988 & 0.003 \\
\hline A1656/Coma & 972 & $970 \pm 22$ & $5.05 \pm 0.62$ & $8.12 \pm 0.56$ & 3.113 & 0.051 \\
\hline NGC 5044 & 156 & $308 \pm 20$ & $0.17 \pm 0.04$ & $0.06 \pm 0.00$ & 1.563 & 0.002 \\
\hline A1736 & 148 & $832 \pm 43$ & $3.05 \pm 0.57$ & $1.95 \pm 0.52$ & 5.738 & 0.631 \\
\hline A3558 & 509 & $902 \pm 27$ & $3.94 \pm 0.53$ & $5.48 \pm 0.15$ & 2.787 & 0.042 \\
\hline A 3562 & 265 & $1029 \pm 41$ & $6.01 \pm 0.94$ & $2.96 \pm 0.29$ & 2.749 & 0.018 \\
\hline A3571 & 172 & $853 \pm 45$ & $3.32 \pm 0.63$ & $7.38 \pm 0.25$ & 2.198 & 0.012 \\
\hline
\end{tabular}

Notes. Number of spectroscopic redshifts, velocity dispersion, dynamical mass, core-corrected X-ray bolometric luminosity, luminosity concentration, and offset between the BCG and X-ray flux-weighted centroid for the whole HIFLUGCS sample of 64 clusters sorted by RA as shown in Table 1 in Zhang et al. (2011). 
Table 1. continued.

\begin{tabular}{|c|c|c|c|c|c|c|}
\hline Name & $n_{\text {gal }}$ & $\sigma\left(\mathrm{km} \mathrm{s}^{-1}\right)$ & $M_{500}\left(10^{14} M_{\odot}\right)$ & $L_{500}^{\text {cor }}\left(10^{44} \mathrm{erg} / \mathrm{s}\right)$ & $c_{\mathrm{L}}$ & $\Delta R / r_{500}$ \\
\hline A1795 & 179 & $791 \pm 41$ & $2.58 \pm 0.48$ & $6.09 \pm 0.17$ & 1.639 & 0.005 \\
\hline A3581 & 83 & $439 \pm 41$ & $0.45 \pm 0.14$ & $0.20 \pm 0.02$ & 1.617 & 0.018 \\
\hline MKW8 & 183 & $450 \pm 25$ & $0.49 \pm 0.10$ & $0.37 \pm 0.04$ & 4.175 & 0.024 \\
\hline RXC J1504.1-0248/RBS1460 & 208 & $888 \pm 47$ & $3.38 \pm 0.64$ & $14.20 \pm 0.95$ & 1.162 & 0.025 \\
\hline A2029 & 202 & $1247 \pm 61$ & $11.00 \pm 1.97$ & $9.55 \pm 0.61$ & 1.451 & 0.008 \\
\hline A2052 & 168 & $590 \pm 35$ & $1.08 \pm 0.22$ & $1.12 \pm 0.05$ & 1.683 & 0.006 \\
\hline MKW3S/WBL564 & 94 & $599 \pm 42$ & $1.13 \pm 0.27$ & $1.66 \pm 0.07$ & 1.644 & 0.038 \\
\hline A2065 & 204 & $1146 \pm 47$ & $8.38 \pm 1.34$ & $5.19 \pm 0.39$ & 2.134 & 0.039 \\
\hline A2063 & 224 & $646 \pm 33$ & $1.42 \pm 0.26$ & $1.36 \pm 0.06$ & 2.259 & 0.010 \\
\hline A2142 & 233 & $1008 \pm 46$ & $5.48 \pm 0.94$ & $9.69 \pm 0.97$ & 2.360 & 0.004 \\
\hline A 2147 & 397 & $859 \pm 32$ & $3.40 \pm 0.52$ & $3.21 \pm 0.47$ & 4.296 & 0.009 \\
\hline A 2163 & 311 & $1498 \pm 61$ & $18.50 \pm 2.95$ & $63.78 \pm 5.24$ & 2.518 & 0.074 \\
\hline A2199 & 374 & $733 \pm 29$ & $2.08 \pm 0.33$ & $2.02 \pm 0.14$ & 1.758 & 0.012 \\
\hline A2204 & 111 & $917 \pm 99$ & $3.90 \pm 1.33$ & $13.94 \pm 0.76$ & 1.384 & 0.006 \\
\hline A2244 & 106 & $1116 \pm 63$ & $7.57 \pm 1.51$ & $5.73 \pm 1.09$ & 1.611 & 0.014 \\
\hline A2256 & 296 & $1216 \pm 45$ & $10.24 \pm 1.53$ & $8.42 \pm 0.39$ & 2.683 & 0.104 \\
\hline A2255 & 189 & $998 \pm 55$ & $5.33 \pm 1.04$ & $5.98 \pm 0.48$ & 4.142 & 0.213 \\
\hline A 3667 & 580 & $1073 \pm 37$ & $6.85 \pm 0.99$ & $8.75 \pm 0.20$ & 3.361 & 0.100 \\
\hline AS1101/Sérsic 159-03 & 20 & $422 \pm 55$ & $0.40 \pm 0.17$ & $1.37 \pm 0.06$ & 1.627 & 0.009 \\
\hline A2589 & 94 & $762 \pm 57$ & $2.32 \pm 0.58$ & $1.14 \pm 0.07$ & 1.969 & 0.008 \\
\hline A2597 & 44 & $525 \pm 54$ & $0.74 \pm 0.24$ & $2.75 \pm 0.11$ & 1.533 & 0.011 \\
\hline A2634 & 192 & $721 \pm 38$ & $1.98 \pm 0.37$ & $0.74 \pm 0.07$ & 4.825 & 0.003 \\
\hline A2657 & 64 & $764 \pm 92$ & $2.34 \pm 0.88$ & $1.54 \pm 0.12$ & 2.325 & 0.018 \\
\hline A4038 & 202 & $764 \pm 37$ & $2.36 \pm 0.42$ & $0.99 \pm 0.08$ & 1.822 & 0.010 \\
\hline A4059 & 188 & $674 \pm 43$ & $1.60 \pm 0.35$ & $1.70 \pm 0.09$ & 1.910 & 0.014 \\
\hline
\end{tabular}

Table 2. Ratios of X-ray bolometric luminosity measurements in different annuli for the whole HIFLUGCS sample of 64 clusters.

\begin{tabular}{lrrrrr}
\hline \hline Luminosity ratios & Mean & Maximum & Minimum & Stddev & Median \\
\hline$L\left(0.2 r_{500} \leq R \leq r_{500}\right) / L\left(0.2 r_{500} \leq R \leq 2.5 r_{500}\right)$ & 0.749 & 0.950 & 0.529 & 0.093 & 0.760 \\
$L\left(R \leq r_{500}\right) / L\left(R \leq 2.5 r_{500}\right)$ & 0.842 & 0.968 & 0.594 & 0.088 & 0.863 \\
$L\left(0.2 r_{500} \leq R \leq r_{500}\right) / L\left(R \leq r_{500}\right)$ & 0.543 & 0.826 & 0.140 & 0.158 & 0.539 \\
$L\left(0.2 r_{500} \leq R \leq 2.5 r_{500}\right) / L\left(R \leq 2.5 r_{500}\right)$ & 0.606 & 0.886 & 0.189 & 0.161 & 0.615 \\
\hline
\end{tabular}

Pratt et al. 2009). Different methods in deriving the X-ray luminosity may lead to different normalization values of the $L-M$ relation and also to different slopes. We therefore tabulate the ratios of the X-ray luminosity derived within different annuli for the whole HIFLUGCS of 64 clusters in Table 2. These values help to ensure a fair comparison between our results and those in other papers. Since the X-ray luminosity varies very little with the truncation radius, the correlation introduced by using the dynamical mass-determined $r_{500}$ in deriving the X-ray luminosity to the $L^{\text {cor }}-M$ relation is negligible.

\subsection{Optical data and analysis}

The positions of the brightest cluster galaxies (BCGs) for 63 clusters are listed in Table 1 in Zhang et al. (2011) and for RXC J1504.1-0248 in Zhang et al. (2012). We obtained the velocity of the cluster galaxies from the literature (updated until
April 2013, including the compilation in Andernach et al. 2005; and Zhang et al. 2011, 2012). Since the individual error estimates are inhomogeneous, we decided not to weight the calculation of the average velocity. When there was more than one velocity per galaxy available, we calculated an average of the measurements, excluding discordant values and those with large errors.

We checked recent redshift surveys and found significant (>90) numbers of new redshifts for Abell clusters: A2029 from Tyler et al. (2013), A2142 from Owers et al. (2011), A2255 from Tyler et al. (2014), and A2256 from Rines et al. (2016). For 2A 0335+96, NED now offers more redshifts from Huchra et al. (2012), resulting in 14 galaxies between 8400 and $13200 \mathrm{~km} \mathrm{~s}^{-1}$, with a velocity dispersion of about $720 \mathrm{~km} \mathrm{~s}^{-1}$. For NGC 1550, we found 42 galaxies with a velocity dispersion of about $700 \mathrm{~km} \mathrm{~s}^{-1}$.

This work focuses on calibrating the uncertainties of the redshift and dynamical mass estimates when only reduced numbers 
of galaxy redshifts per cluster are used. The effect on our study by adding the new redshifts mentioned above is negligible. We therefore decided to stay with our compilation without updating it. In our upcoming study, we aim to follow up clusters with low numbers of spectroscopic members to ensure more than about 45 members for all clusters in the sample so that we can derive reliable mass estimates for all individual clusters and calibrate the relation of luminosity versus mass by taking the sample selection effect into account, that is, the Malmquist bias (Malmquist 1922) that arises from working with a flux-limited sample. We shall therefore revise the dynamical mass estimates, including the new redshifts mentioned above.

\subsubsection{Cluster galaxy selection}

In hierarchical structure-formation scenarios, the spherical infall model predicts a trumpet-shaped region in the diagram of lineof-sight velocity versus projected distance, the so-called caustic (e.g., Kaiser 1987). The boundary of the caustic defines galaxies inside as cluster galaxies and those outside as fore- and background galaxies. For each cluster, we plotted the line-of-sight velocity of the selected galaxies as a function of their projected distance from the BCG and located the caustic that efficiently excludes interlopers (e.g., Diaferio 1999; Katgert et al. 2004; Rines $\&$ Diaferio 2006). We considered only the galaxies inside the caustic as cluster galaxies and excluded the others from the subsequent analysis. We list the number of cluster galaxies $\left(n_{\text {gal }}\right)$ for the HIFLUGCS in Table 1. We gathered redshifts of a total of 13650 cluster galaxies after the caustic member selection. Since 2A $0355+096$ has only three redshifts, we excluded it from the study involving cluster redshift and dynamical mass estimates. The median number of spectroscopic members for the remaining sample is 188 per cluster.

There are another six clusters that have fewer than 45 cluster galaxies with spectroscopic redshifts in the HIFLUGCS (i.e., 13 galaxies for A0478, 22 for NGC1550, 42 for EXO 0422-086, 37 for Hydra A, 20 for S1101, and 44 for A2597), which were maintained in the redshift and dynamical mass study. All six systems with $<45$ cluster galaxy redshifts are $\mathrm{CC}$ clusters. The reason for having $<45$ cluster galaxy redshifts is that these clusters are not covered by large spectroscopic surveys such as SDSS or $2 \mathrm{dF} / 6 \mathrm{dF}$. We aim to observe them with ground-based telescopes. Recently, we obtained new redshifts from VLT/VIMOS for Abell S1101 (Rabitz et al. 2017).

\subsubsection{Redshift, dynamical mass, and cluster radius}

We applied the bi-weight estimator (e.g., Beers et al. 1990) to the cluster galaxies to measure the cluster redshift $(z)$ and velocity dispersion $(\sigma)$. The errors were estimated through 1000 bootstrap simulations.

The caustic method mentioned in Sect. 2.2.1 can provide an estimate of the escape velocity and thus the matter distribution for the halo according to the distribution of the caustic amplitude (e.g., Diaferio 1999). However, only with a large number of spectroscopic members, for instance, 200 members, are we likely to recover a rather complete sampling of the caustic, which yields a reliable measurement of the underlying dark matter distribution of the cluster, as shown, e.g., by the simulations in Serra \& Diaferio (2013), for instance. Their simulations also showed that fewer members tend to provide a slightly reduced amplitude of the caustic because the caustic is undersampled, which causes underestimation of the total mass.
To optimize the optical spectroscopic follow-up for galaxy clusters, particularly at high redshifts where the cluster galaxy member statistic is low, we focused on the method for deriving the dynamical mass based on a small number of cluster galaxies, which differs from the caustic method. There are a number of such methods that are calibrated by samples of simulated clusters (e.g., Biviano et al. 2006; Munari et al. 2013). We note that the difference between the dynamical masses derived with these methods is rather small, which is within the uncertainties of our dynamical mass estimates. In addition, we focused only on the relative variation in the dynamical mass measurements that is normalized to the input dynamical mass in the investigations of the systematic bias and uncertainties of redshift and dynamical mass estimates based on MC resampling (Sect. 4).

Our method of mass estimation is based entirely on the velocity dispersion, as explained in Sect. 3 of Biviano et al. (2006, $M_{\sigma}$ therein). We followed the Navarro et al. (NFW, 1997) model used in Biviano et al. (2006) to derive $M_{500}$ from $M_{\sigma}$. The dimensionless Hubble parameter, $E(z)=\sqrt{\Omega_{\mathrm{m}}(1+z)^{3}+\Omega_{\Lambda}}$, was added to their formulation since not all our clusters are at $z \sim 0$. We used the dynamical mass at the radius within which the overdensity was 500 times the critical density as the cluster mass $\left(M_{500}\right.$, Table 1$)$. We derived the cluster radius $\left(r_{500}\right)$ from the dynamical mass $\left(M_{500}\right)$, different from the method used by Zhang et al. (2011).

\subsection{Cluster morphology}

Although the cluster properties are driven by gravity, predominantly from dark matter, the baryon physics on small scales modifies the scaling relation from the self-similar prediction through cooling, merging, and feedback from star formation and AGN activities. We divided the HIFLUGCS into undisturbed versus disturbed subsamples as well as CC and NCC subsamples to study the effect of mergers and CC clusters, respectively, on our results.

\subsubsection{Undisturbed versus disturbed clusters}

Many studies found that a large offset between the BCG and $\mathrm{X}$-ray peak/centroid probably indicates that more merging has occurred in the past (e.g., Katayama et al. 2003; Hudson et al. 2010; Zhang et al. 2010, 2011). We converted the angular separation between the X-ray flux-weighted center and BCG position into the physical separation at the cluster redshift for all 64 clusters in the HIFLUGCS. As shown in Zhang et al. (2011), the offset in projection between the X-ray flux-weighted center and BCG position scaled by the cluster radius, $\Delta R / r_{500}$, serves very well to separate the disturbed from the undisturbed clusters.

The luminosity concentration is also one of the parameters suitable to separate the clusters (e.g., Santos et al. 2008). Here we set this parameter to be $c_{\mathrm{L}}=L\left(R \leq r_{500}\right) / L\left(R \leq 0.2 r_{500}\right)$, and we integrated the surface brightness within a projected clustercentric distance of $r_{500}$ and $0.2 r_{500}$ to derive the bolometric X-ray luminosity of $L\left(R \leq r_{500}\right)$ and $L\left(R \leq 0.2 r_{500}\right)$, respectively. All HIFLUGCS clusters also have Chandra data, but mainly in the field of the cluster cores, not even out to $0.2 r_{500}$ for a number of cases. We therefore preferred to rely on XMM-Newton data to compute the $c_{\mathrm{L}}$ parameter.

In the following we combine the cuts of $c_{\mathrm{L}}$ and $\Delta R / r_{500}$ to effectively separate the sample into undisturbed and disturbed clusters. As shown in Fig. 1 and Table 3, the histograms of both $\log _{10} c_{\mathrm{L}}$ and $\log _{10}\left(\Delta R / r_{500}\right)$ of the 64 clusters follow approximately Gaussian distributions. The best power-law fit 

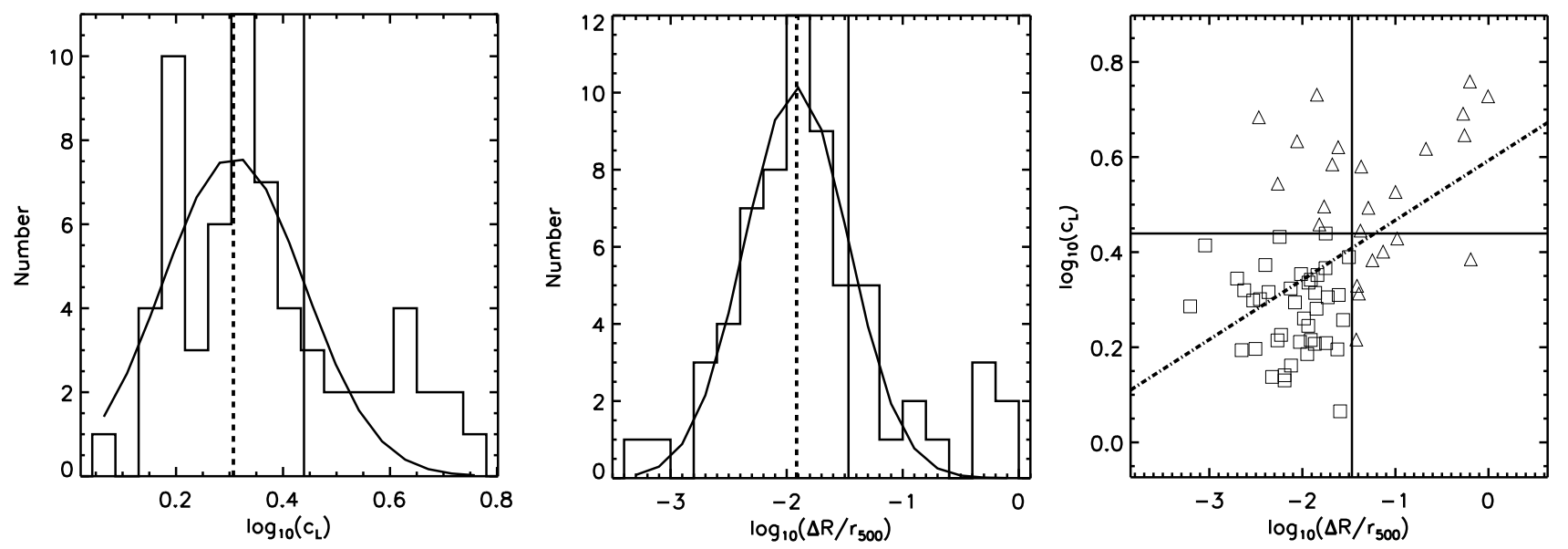

Fig. 1. Left and middle panels: histograms of the luminosity concentration (left) and the offset between the BCG and X-ray flux-weighted centroid (middle) of all 64 HIFLUGCS clusters with their Gaussian fits shown as solid curves. The mean of the Gaussian fits and their $1 \sigma$ clipping are shown as dashed and solid vertical lines, respectively. Right panel: $c_{\mathrm{L}}$ versus $\Delta R / r_{500}$ with its best fit, $\log _{10} c_{\mathrm{L}}=(0.593 \pm 0.053)+(0.125 \pm$ $0.028) \log _{10}\left(\Delta R / r_{500}\right)$, as dash-dotted line. The vertical and horizontal solid lines denote the $1 \sigma$ clipping below which the clusters are considered as undisturbed ones (open squares). The disturbed clusters are shown as open triangles.

Table 3. Gaussian fit parameters of the histograms of the logarithmic values of $c_{\mathrm{L}}$ and $\Delta R / r_{500}$ for the full HIFLUGCS sample of all 64 clusters.

\begin{tabular}{lrr}
\hline \hline Distribution & Mean & $F W H M$ \\
\hline $\log _{10} c_{\mathrm{L}}$ & $0.31 \pm 0.03$ & $0.13 \pm 0.03$ \\
$\log _{10}\left(\Delta R / r_{500}\right)$ & $-1.91 \pm 0.04$ & $0.45 \pm 0.04$ \\
\hline
\end{tabular}

between the two parameters for the 64 clusters is $\log _{10} c_{\mathrm{L}}=$ $(0.593 \pm 0.053)+(0.125 \pm 0.028) \log _{10}\left(\Delta R / r_{500}\right)$. We divided the whole sample into disturbed and undisturbed clusters using the $1 \sigma$-clipping of the Gaussian-mean values of these two histograms. Those with low values of both $c_{\mathrm{L}}$ and $\Delta R / r_{500}$ are considered as undisturbed clusters (see the right panel of Fig. 1). The cuts divide the sample into 39 undisturbed clusters and 24 disturbed clusters.

\subsubsection{CC versus NCC clusters}

The relation of gas mass versus total mass in recent work (e.g., Arnaud et al. 2005; Zhang et al. 2008) shows a slope shallower than unity, the self-similar prediction, which may be accounted for by the mass dependence of the gas mass fraction in $\mathrm{CC}$ and NCC systems (e.g., Eckert et al. 2012). Therefore, we also investigated our results for the subsamples of CC and NCC clusters, respectively.

The central cooling time can be accurately estimated from Chandra data because of its smaller point-spread function (PSF). We used the central cooling time calculated at $0.004 r_{500}$ from Eq. (15) in Hudson et al. (2010) and divided the sample of the 64 clusters into $28 \mathrm{CC}$ clusters and $36 \mathrm{NCC}$ clusters as listed in Table 2 in Zhang et al. (2011).

\section{X-ray luminosity versus dynamical mass}

We carried out the X-ray and optical analyses independently, in addition to taking the dynamical mass-determined $r_{500}$ in computing the X-ray bolometric luminosity. Since the luminosity values measured within $r_{500}$ and $2.5 r_{500}$ differ only by $\sim 15 \%$ on average, as shown in Table 2 , using the cluster radius, $r_{500}$, derived from the dynamical mass to compute the X-ray bolometric luminosity is not expected to cause any significant bias in our result.

We fit the $L^{\text {cor }}-M$ relation by a power-law,

$\log _{10} \widetilde{L}=A \log _{10} \widetilde{M}+B$,

in which $\widetilde{L}=\frac{L^{\text {cor }}}{E(z) 10^{44} \mathrm{erg} / \mathrm{s}}$ and $\widetilde{M}=\frac{M E(z)}{10^{14} M_{\odot}}$. We note that the HIFLUGCS is a flux-limited sample, which consists of clusters covering a broad range of redshifts (i.e., from $z=0.0037$ to $z=0.2153)$. Therefore, we included the $E(z)$ factor in the scaling relation to account for the evolution of the geometry of the Universe in this redshift range. We assumed a constant standard deviation of the luminosity from this relation in logarithmic scale and used $\sigma_{\log _{10} L}$ for the intrinsic scatter of the luminosity in logarithmic scale. We chose the relatively simple algorithm given in Akritas \& Bershady (1996) to perform the fit to the relation $y=A x+B$, in which

$\chi^{2}=\sum_{i} \frac{\left(y_{i}-A x_{i}-B\right)^{2}}{\sigma_{y, i}^{2}+A^{2} \sigma_{x, i}^{2}}$.

The scatter of $y$ consists of two components, the statistical and intrinsic scatter, which satisfy $\sigma_{y, i}^{2}=\sigma_{y, i, \text { sta }}^{2}+\sigma_{y, i, \text { int }}^{2}$ (e.g., Weiner et al. 2006). In Table 4 we list the best-fit parameters and also include the normalization values by setting the slope to the selfsimilar prediction, namely $4 / 3$. The data still agree with a slope of $4 / 3$ within $2 \sigma$ uncertainties of the best-fit slope parameters.

\section{1. $L^{\text {cor }}-M$ relation for the $n_{\text {gal }} \geq 45$ clusters}

The dynamical masses tend to be underestimated for clusters with too few redshift measurements according to the clusters in simulations (e.g., Biviano et al. 2006; Zhang et al. 2011). The effect becomes negligible (i.e., on a level of a few per cent) when clusters with a sufficient number of spectroscopic cluster galaxies are considered.

Zhang et al. (2011) found that the velocity dispersion measurements, and thus the dynamical masses, may be significantly 
Y.-Y. Zhang et al.: HIFLUGCS: $L-M$ relation and its implications for mass calibrations with the SPIDERS and 4MOST surveys
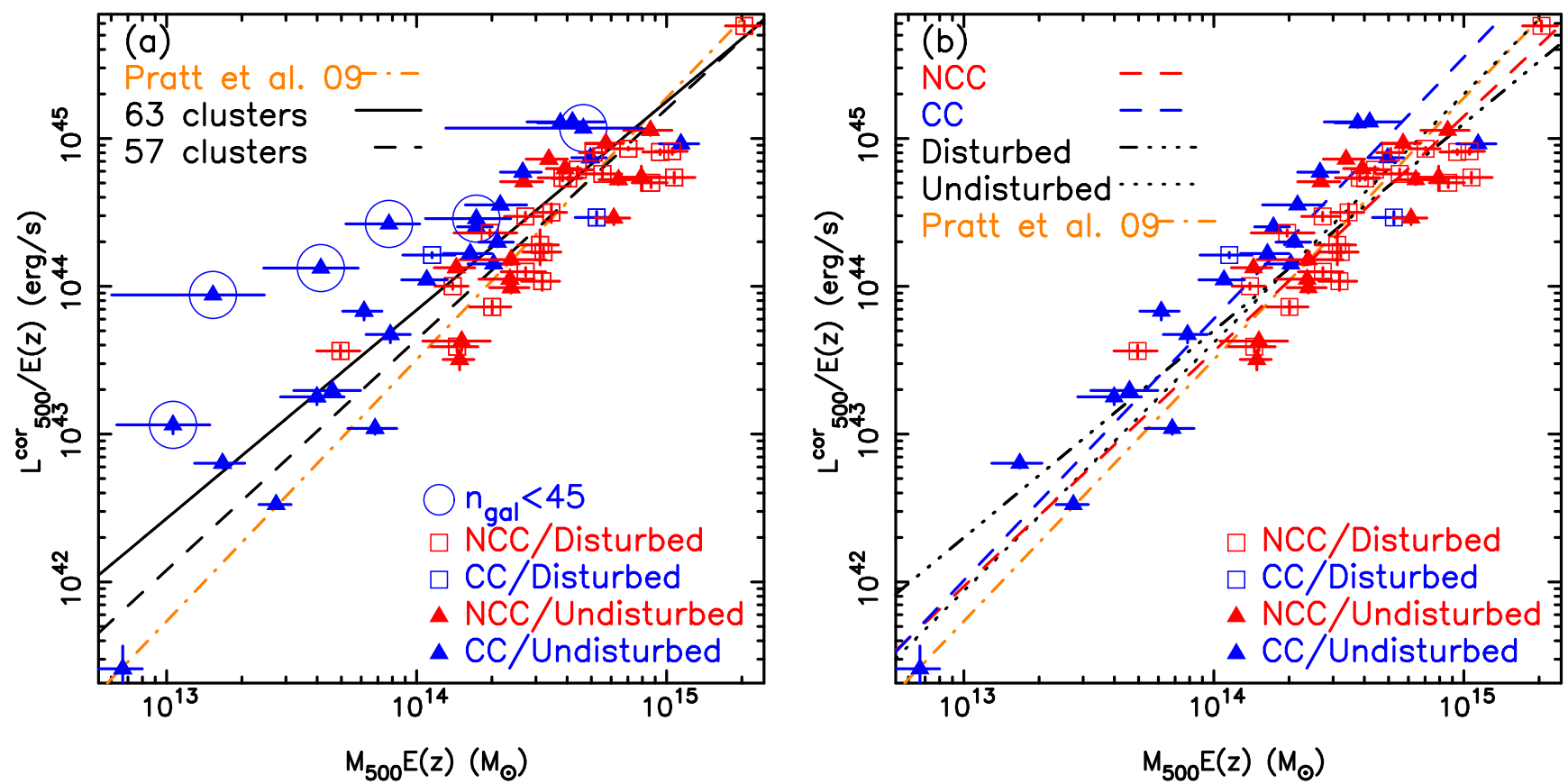

Fig. 2. a) Core-corrected bolometric X-ray luminosity versus dynamical mass for the 63 clusters with the best power-law fits for the 63 and $57\left(n_{\text {gal }} \geq 45\right)$ clusters as black solid and dashed lines, respectively. b) Core-corrected X-ray bolometric luminosity versus dynamical mass for the $57\left(n_{\text {gal }} \geq 45\right)$ clusters with the best power-law fits for the NCC, CC, disturbed, and undisturbed clusters as red dashed, blue dashed, black dot-dot-dot-dashed, and black dotted lines, respectively.

Table 4. Best power-law fits, $\log _{10} \widetilde{L}=A \log _{10} \widetilde{M}+B$, in which $\widetilde{L}=\frac{L^{\text {cor }}}{E(z) 10^{44} \mathrm{erg} / \mathrm{s}}$ and $\widetilde{M}=\frac{M E(z)}{10^{14} M_{\odot}}$, of the $L^{\text {cor }}-M$ relations.

\begin{tabular}{llllll}
\hline \hline Sample & $\begin{array}{l}\text { Number } \\
\text { of clusters }\end{array}$ & $B$ & $A$ & $\sigma_{\log _{10} L \text {,intrinsic }}$ & $B$ for $A=4 / 3$ \\
& 63 & $-0.16 \pm 0.06$ & $1.29 \pm 0.09$ & $0.33 \pm 0.04$ & $-0.18 \pm 0.04$ \\
Whole & 39 & $-0.14 \pm 0.07$ & $1.36 \pm 0.13$ & $0.39 \pm 0.05$ & $-0.12 \pm 0.06$ \\
Undisturbed & 24 & $-0.29 \pm 0.10$ & $1.26 \pm 0.14$ & $0.22 \pm 0.03$ & $-0.28 \pm 0.05$ \\
Disturbed & 27 & $+0.02 \pm 0.08$ & $1.45 \pm 0.16$ & $0.41 \pm 0.07$ & $-0.04 \pm 0.08$ \\
$\mathrm{CC}$ & 36 & $-0.27 \pm 0.09$ & $1.41 \pm 0.13$ & $0.23 \pm 0.02$ & $-0.28 \pm 0.04$ \\
NCC & 57 & $-0.35 \pm 0.05$ & $1.44 \pm 0.08$ & $0.27 \pm 0.02$ & $-0.24 \pm 0.04$ \\
\hline Whole $\left(n_{\text {gal }} \geq 45\right)$ & 33 & $-0.28 \pm 0.06$ & $1.57 \pm 0.11$ & $0.29 \pm 0.03$ & $-0.21 \pm 0.06$ \\
Undisturbed $\left(n_{\text {gal }} \geq 45\right)$ & 21 & $-0.16 \pm 0.07$ & $1.66 \pm 0.13$ & $0.29 \pm 0.06$ & $-0.16 \pm 0.08$ \\
CC $\left(n_{\text {gal }} \geq 45\right)$ & & & & & \\
\hline
\end{tabular}

Notes. All disturbed clusters as well as NCC clusters have at least 45 member galaxies with spectroscopic redshifts per cluster in the current observational sample.

underestimated for clusters with fewer than 45 spectroscopic member galaxies in the sample. To avoid any bias due to extreme outliers with too few cluster galaxies with spectroscopic redshifts, we show the $L^{\text {cor }}-M$ relations for the 63 clusters (Fig. 2a) and for the 57 clusters that have at least 45 cluster galaxies with spectroscopic redshifts (Fig. 2b), respectively. For the 63 clusters, the slope, $1.29 \pm 0.09$, agrees with the self-similar prediction. However, most recent work shows steeper slopes, such as $1.77 \pm 0.07$ in Pratt et al. (2009). Taking a close look, the systems with very few redshifts appear to cause the shallow slope. In contrast to that of the 63 clusters, the slope of the 57 clusters is steeper. The selection effect, that is, the Malmquist bias that should be corrected for a flux-limited sample, should lead to an even steeper slope for the 57 clusters, which tends to agree better with recent X-ray calibrated $L-M$ relations (e.g., Pratt et al. 2009). At the same time, the fact that the six discarded clusters are all CC clusters may also have an effect on the total best-fit slope, although there appears little difference between the slopes of the $L-M$ relations between CC and NCC clusters.

\section{2. $L^{\text {cor }}-M$ relations for the CC/NCC and undisturbed/disturbed subsamples}

We list the best-fit $L^{\text {cor }}-M$ relations for the different classes of clusters in Table 4. As shown in Figs. 2b and 3, the NCC clusters are undersampled in the low-mass regime since the HIFLUGCS is a flux-limited sample. The slope parameters agree within $1 \sigma$ error between the undisturbed and disturbed clusters as well as between the CC and NCC clusters. There is no obvious mass dependence in the scatter of the core-to-total luminosity ratio $\left(1 / c_{\mathrm{L}}\right)$ for the CC and NCC subsamples, respectively, as shown in Fig. 3. The $L^{\text {cor }}-M$ relation for the disturbed clusters has smaller intrinsic scatter than that for the undisturbed clusters. 


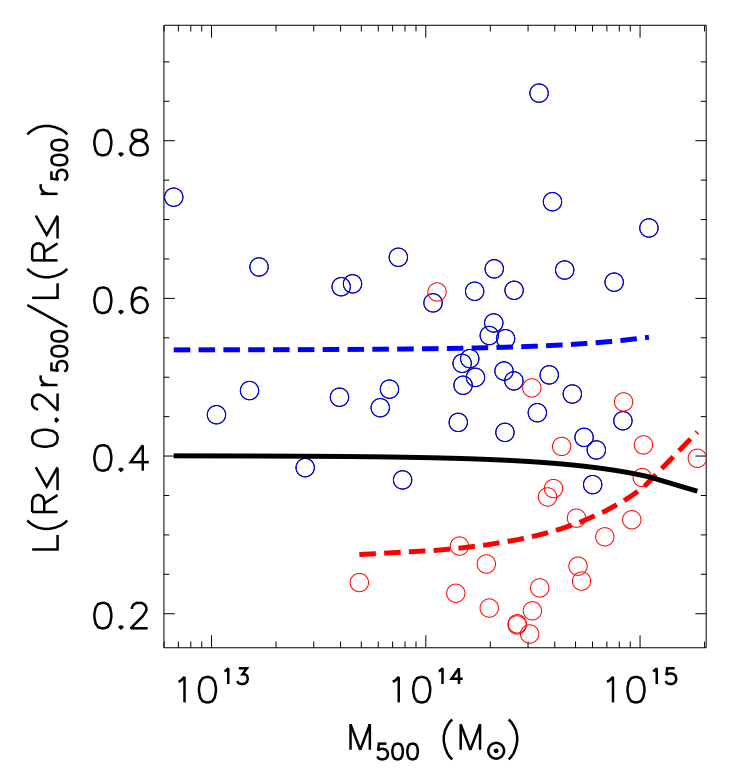

Fig. 3. X-ray core-to- $r_{500}$ luminosity fraction versus dynamical mass for the CC (blue) and NCC (red) clusters. The curves show the regression fits for all (black), CC (blue) and NCC (red) clusters, respectively. There is no obvious dependence considering the observed scatter.

The intrinsic scatter of the NCC clusters is also smaller than that of the CC clusters even when the core-corrected luminosity is used. This partially depends on how broad the cut is that was used to classify the corresponding cluster subsamples. The normalization of the disturbed (NCC) clusters agrees better with the X-ray calibrated relation (e.g., Zhang et al. 2008, see Table 3 therein; Pratt et al. 2009). Assuming a fixed slope of $4 / 3$, the normalization agrees between different cluster types within the uncertainties.

A comparison of the best fits for the undisturbed (CC) clusters in the samples of the 57 and 63 clusters in Fig. 2 clearly shows that the scatter for the undisturbed (CC) clusters in the sample of 57 clusters with $N_{\text {gal }}>45$ is lower than that for the sample of 63 clusters. As shown in Fig. $2 b$ for the 57 clusters, the normalization on average agrees within $17 \%$ between the undisturbed and disturbed clusters, and within $32 \%$ between the CC and NCC clusters. The slopes still agree among the subsamples. As the scatter is dominated by the CC clusters, the scatter of the $L^{\text {cor }}-M$ relation may mainly be due to the integrated effect of the structure formation history instead of recent extreme merging.

\section{Systematic bias and uncertainties of redshift and dynamical mass estimates based on MC resampling}

The results from our sample show that clusters with only few spectroscopic members tend to have underestimated dynamical masses. It is therefore of prime importance to quantify any systematic bias and uncertainties of the dynamical mass estimate and their implications on mass calibrations using upcoming optical spectroscopic surveys.

Both large optical spectroscopic surveys and individual pointed observations will be used to follow up galaxy clusters detected in upcoming large X-ray surveys such as eROSITA. The huge amount of optical spectroscopic follow-up data might be combined with the X-ray data for mass calibrations, in particular, in different redshift ranges. This allows constraining the evolution of the mass scaling relations, which becomes important when using high-redshift clusters for cosmology. In the following, we calculate the cluster redshift and estimate the dynamical mass using subsamples of member galaxies that we resampled according to eight optical spectroscopic setups, which demonstrates their ability for studying mass calibrations using the follow-up of the X-ray detected clusters. We note that the resampled galaxies were selected from the catalog of the causticbounded galaxies. Therefore, the probability of contamination from interlopers is low. The systematic uncertainties are mainly caused by statistical fluctuations in the resampling. Our study is also useful to assign the mass uncertainty in the stacking analysis using cluster samples with a rather clean member selection but a small number of members (e.g., Clerc et al. 2016).

\subsection{Optical spectroscopic survey setups}

The extended Baryon Oscillation Spectroscopic Survey (eBOSS; e.g., Schlegel et al. 2011) is a redshift survey covering a wavelength range from $340 \mathrm{~nm}$ to $1060 \mathrm{~nm}$, with a resolution $R=3000-4800$. This survey targets objects up to redshift 2. With the eBOSS setup, the SPectroscopic IDentification of ERosita Sources (SPIDERS; e.g., Merloni et al. 2012) survey has been designed to follow up X-ray selected active galactic nuclei (AGN) and clusters over $\sim 7500$ degree $^{2}$ area. Before eROSITA data will be available, SPIDERS is targeting ROSAT and XMM-Newton sources (see Clerc et al. 2016; Dwelly et al., in prep.). In the later years of operations, SPIDERS will target eROSITA sources detected in the first two years of observations (see Merloni et al. 2012). The 4-m Multi-Object Spectroscopic Telescope (4MOST, e.g., de Jong et al. 2012) is designed to obtain $>1$ million redshifts for $>50000$ clusters in the southern sky in the eROSITA survey. The Euclid mission can follow up high-redshift galaxies through their emission lines (e.g., Laureijs et al. 2011; Pointecouteau et al. 2013). In this study, however, we do not discuss the optimization of the follow-up of the Euclid survey since Euclid provides slitless spectroscopy, which has no fiber constraint. Euclid will instead focus on how to improve the situation due to overlapping spectra in densely populated regions for optimizing its strategy, for example.

In practice, only several cluster galaxies per cluster can be targeted because of the fiber spacing constraint for multi-object spectroscopic surveys; this is not the case for slitless spectroscopic surveys like Euclid. Moreover, toward the high-redshift regime, a bright limiting magnitude cut can strongly limit the number of spectroscopic members. To separate the two effects, we performed the investigations in two steps: (i) for the setups using the closest fiber separation alone and (ii) for the setups including constraints from both the closest fiber separation and limiting magnitude cuts. SPIDERS uses a minimum fiber separation of $65^{\prime \prime}$. To demonstrate the effect of the fiber separation, we used a fiber spacing constraint of 55" and $65^{\prime \prime}$, respectively, in our MC simulations for SPIDERS. The limiting magnitude is the fiber magnitude. Toward the very high-redshift regime, optical spectroscopy of several of the bright galaxies already requires high-sensitivity optical spectrographs, for instance, on the Very Large Telescope (VLT). We also investigated the setups with a limited number of cluster redshifts. Below are the eight setups in which the BCG was always first selected for targeting.

(I) SPIDERS_55: it has a closest fiber separation of 55". Each position can be pointed only once. 
(II) SPIDERS_65: it has a closest fiber separation of $65^{\prime \prime}$. Each position can be pointed only once.

(III) 4MOST: it has a closest fiber separation of 20". Each position can be pointed four times.

(IV) SPIDERS_55m: it has a closest fiber separation of 55". Each position can be pointed only once. The limiting magnitude is $i_{\mathrm{AB}}=21$.

(V) SPIDERS_65 m: it has a closest fiber separation of $65^{\prime \prime}$. Each position can be pointed only once. The limiting magnitude is $i_{\mathrm{AB}}=21$.

(VI) 4MOSTm: it has a closest fiber separation of $20^{\prime \prime}$. Each position can be pointed four times. The limiting magnitude is $r_{\mathrm{AB}}=22$.

(VII) 10zs: it samples the BCG and nine randomly selected remaining member galaxies per cluster.

(VIII) 05zs: it samples the BCG and four randomly selected remaining member galaxies per cluster.

\subsection{Input cluster redshifts, $z_{\text {in }}$, in the $I-V I$ setups}

Follow-up of high-redshift systems is particularly important to constrain the evolution of mass calibrations, which is still poorly understood. Technically, nearby clusters can tolerate high values of the closest fiber separation in the optical spectroscopy. The angular size of a cluster decreases toward high redshift. The number of possible spectroscopic targets decreases with redshift given a fixed value of the closest fiber separation. Therefore, we tested the I-VI setups assuming input cluster redshifts at the HIFLUGCS cluster redshifts ( $z$ in Table 1 ) as well as placing the clusters at the redshift values of $0.2,0.4,0.6$, and 0.8 , respectively. We note that the relative rest-frame line-of-sight velocity of any member galaxy to its host cluster was not modified in this process.

\subsection{Limiting magnitude cuts in the IV-VI setups}

We included the limiting magnitude cuts in resampling the member galaxies in the IV-VI setups as follows.

\subsubsection{Shape of the galaxy luminosity function (GLF)}

We assumed that the observed spectroscopic member galaxies are those at the bright end of the GLF. We adopted the Schechter function fit of the GLF of the bright galaxies within a projected cluster-centric distance of $2 \mathrm{Mpc}$ in the $i$ and $r$ bands (Popesso et al. 2005, Table 2, local background) for the SPIDERS and 4MOST surveys, respectively. Based on the results in Hansen et al. (2009), we assumed that the shape of the GLF of the bright red members does not change with cluster-centric radius for simplicity.

\subsubsection{Normalization of the GLF}

Several richness versus mass calibrations have been reported (e.g., Hansen et al. 2005, 2009; Johnston et al. 2007; Reyes et al. 2008). We took the richness versus weak-lensing mass calibration given by Eq. (10) in Hansen et al. (2009), in which the masses are measured within the radius where the mass overdensity is 200 relative to the critical density, and calculated the richness from the cluster dynamical mass for each HIFLUGCS cluster.

For simplicity, we assumed that the red member galaxies brighter than $\left(i^{*}+1\right)$ are similar to those brighter than $\left(r^{*}+1\right)$.
Therefore, the number of the red member galaxies brighter than $\left(i^{*}+1\right)$ is similar to the number of the red members brighter than $\left(r^{*}+1\right)$. We obtained the richness by integrating the GLF down to $\left(i^{*}+1\right)$ magnitude for the SPIDERS survey and $\left(r^{*}+1\right)$ magnitude for the 4MOST survey, respectively. Assuming the richness obtained from the dynamical mass equal to the richness derived by integrating the GLF, we obtained the normalization of the GLF.

\subsubsection{Galactic extinction and $K$-corrections}

We corrected apparent magnitudes for Galactic extinction using the maps of Schlegel et al. (1998). We assumed that photometric errors at bright magnitudes are 0.05 . We used typical colors and their color uncertainties of luminous red galaxies of Maraston et al. (2009), and applied $K$-correction and evolutionary correction using the luminous red galaxy template in kcorrect (v4_2, Blanton \& Roweis 2007).

\subsubsection{Member selection}

The maximum number of member galaxies, $n_{\max }$, that can be spectroscopically followed-up was computed by integrating the GLF down to the limiting magnitude. In Sect. 4.3.1 we assumed that the observed spectroscopic member galaxies are those filling in the bright end of the GLF. Therefore, if the number of cluster members for the HIFLUGCS clusters $\left(n_{\text {gal }}\right.$ in Table 1$)$ is larger than $n_{\text {max }}$, then the fraction of the $n_{\text {gal }}$ spectroscopic members that are brighter than the limiting magnitude is $f_{\mathrm{obs}}=n_{\mathrm{max}} / n_{\mathrm{gal}}$; otherwise, all existing spectroscopic members are brighter than the limiting magnitude, $n_{\max }=n_{\text {gal }}$.

Many cluster members in the observational sample have no $i$ and $r$ magnitudes from the NED. As a preliminary selection, we first took the BCG and then randomly took $f_{\text {obs }} n_{\text {gal }}-1$ additional spectroscopic members using the MC method. The initially selected members were filtered further according to the values of the closest fiber separation.

\subsubsection{Effect of flux loss of the aperture magnitude}

The angular sizes of very bright cluster galaxies in the nearby Universe (i.e., $z<0.4$ ) are usually larger than the fiber aperture. The flux-loss correction may become important in deriving the apparent magnitudes from the measured flux in the fiber aperture when considering the limiting magnitude cut. Additionally, the PSF affects the measured apparent magnitudes due to convolution of galaxy images caused by the seeing. As shown by our model of the flux loss in targeting galaxies with spectroscopic fibers in Appendix A, this effect is negligible and skipped in our further analyses.

\subsection{Redshift and dynamical mass measurements}

To estimate the average of the systematic uncertainties, we carried out 500 resampling runs per cluster per setup per input cluster redshift $\left(z_{\text {in }}=z, 0.2,0.4,0.6,0.8\right.$ for I-VI setups and $z_{\text {in }}=z$ for VII-VIII setups). In each run, we measured the cluster redshift, velocity dispersion, and dynamical mass following the procedure in Sect. 2.2.2 when the number of resampled members reached ten, $n_{\mathrm{gal}, i} \geq 10$. Otherwise $\left(n_{\mathrm{gal}, i}<10\right)$, we computed the cluster redshift, which is the mean of the member galaxy redshifts, but not the velocity dispersion and dynamical mass.

The Gaussian mean and bi-weight (see Sect. 2.2.2) mean values of the histograms of the redshift and dynamical mass 

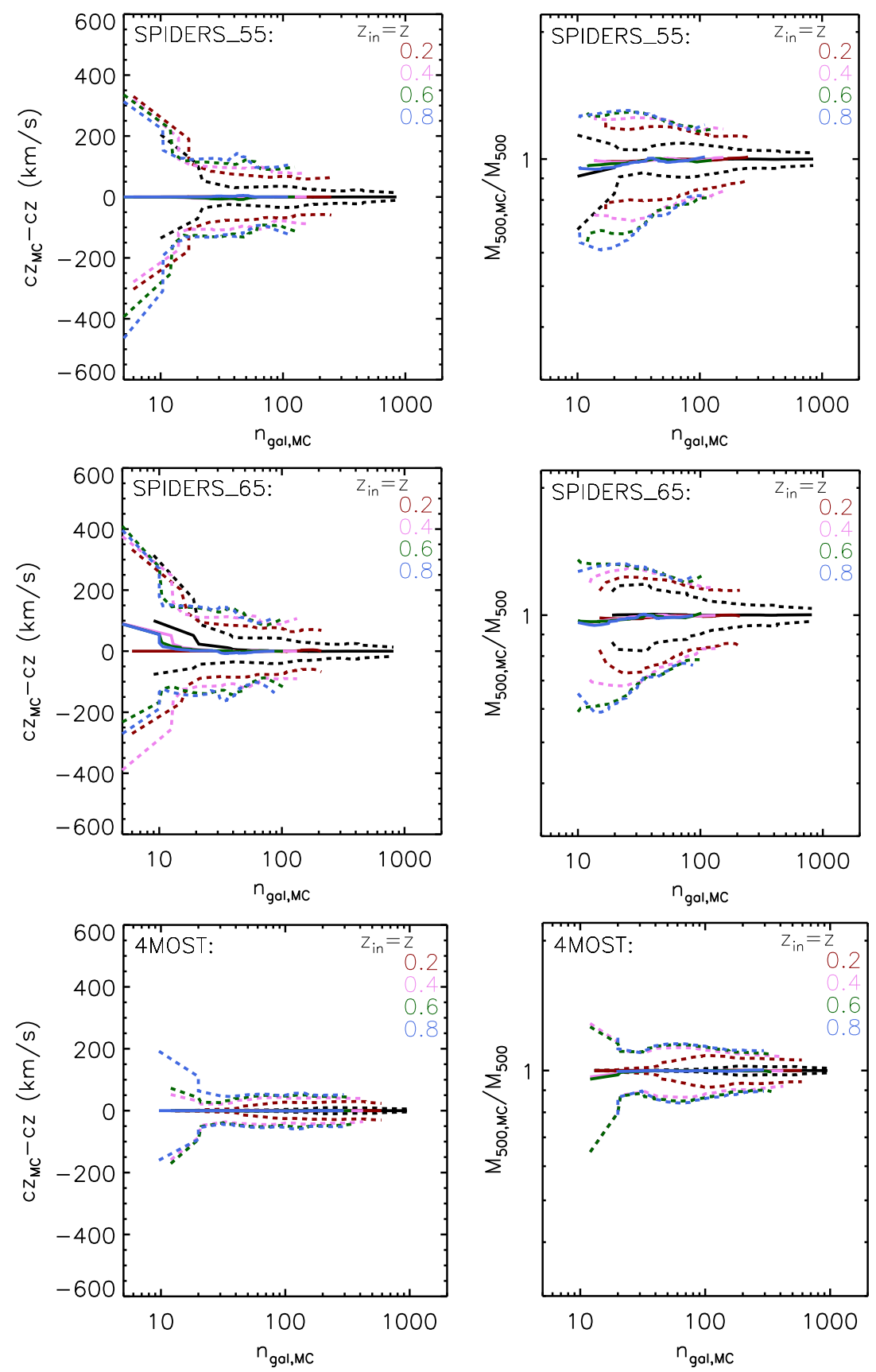

Fig. 4. Bias (solid lines) and dispersion (dashed lines) of the redshift (left panels) and dynamical mass (right panels) measurements in the SPIDERS_55 (upper panels), SPIDERS_65 (middle panels) and 4MOST (lower panels) setups from the input cluster values as a function of the resampled number of redshifts in use. The curves are smoothed with a boxcar average of the specified width of 11 to avoid spikes due to undersampling for a few clusters.

estimates from 500 runs based on the resampled members agree well in all cases except for the 10zs setup. The bi-weight mean of the histograms is in general closer to the input value than the Gaussian mean for the 10zs setup. Therefore, we used the bi-weight mean to calculate the results. The number of member redshifts used per cluster per setup per input cluster redshift, $n_{\text {gal,MC }}$, was computed as the bi-weight mean of $n_{\text {gal }, i}$ with $i=0, \ldots, 499$. As $n_{\mathrm{gal}, i}<10$ may be obtained for some resampling runs, $n_{\text {gal,MC }}$ can be lower than ten for some cases involving the dynamical mass estimates, in which the dynamical mass per cluster per setup per input cluster redshift, $M_{500, \mathrm{MC}}$, is computed as the bi-weight mean of $M_{500, j}$ with $j=i$ if $n_{\text {gal }, i} \geq 10$. Simulated clusters with fewer than ten members were excluded from the mass computation.

\subsection{Summary of results}

\subsubsection{Fraction of selected members}

When we used the collisional distance constraint alone (setups I-III), almost all members were selected in the 4MOST setup, and more than $95 \%$ of the members were selected in the SPIDERS_55 and SPIDERS_65 setups. In the case of ten or five redshifts per cluster, only a small fraction of the members were selected. The fraction of selected members including the limiting magnitude cut (setups IV-VI) decreases faster toward the high-redshift end than that in the resampling assuming only the collisional distance constraint.

As shown in the right panels of Figs. 4-6, the scatter in the dynamical mass estimates for the clusters with at least ten 

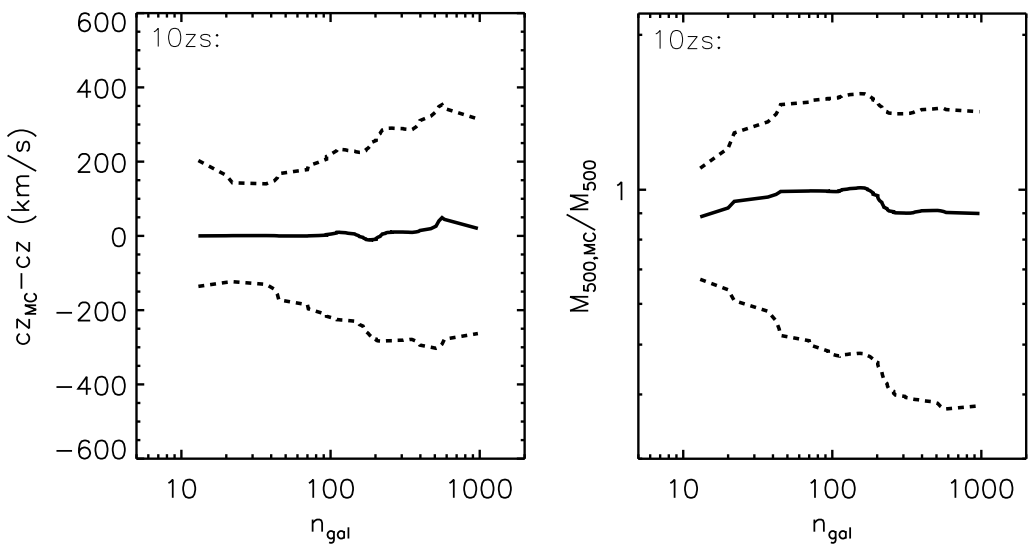

Fig. 5. Bias (solid curves) and dispersion (dashed curves) of the redshift (left panel) and dynamical mass (right panel) measurements in the 10zs setup from the input cluster values versus input number of cluster galaxies. The curves are smoothed with a boxcar average of the specified width of 11 to avoid spikes due to under-sampling for a few clusters. Note that $n_{\text {gal }}$ is the input number of galaxies and the output number of galaxies is always 10 .

members is within $50 \%$, which is the minimum required precision for a stacking analysis for the mass calibration given the known intrinsic scatter in the mass-observable relation (e.g., Biviano et al. 2006). We therefore inspected the fraction of the clusters with at least ten members. All of the 63 clusters in the SPIDERS and 4MOST cases that assume no magnitude cut have more than ten galaxy members per cluster. In the setups with the magnitude limit instead, the fraction of the clusters with at least ten members per cluster decreases with redshift as shown in Fig. 7. In the redshift bins of $0.2,0.4,0.6$, and 0.8 in the SPIDERS_55m setup, there are 1, 3, 8, and 18 clusters out of 63 clusters that have fewer than ten redshifts per cluster. The case for the SPIDERS_65m setup, which uses a larger fiber separation $\left(65^{\prime \prime}\right)$, is slightly worse. In the redshift bins of $0.4,0.6$ and 0.8 using the 4MOSTm setup, there are 1, 2, and 4 clusters out of 63 clusters that have fewer than ten redshifts per cluster.

\subsubsection{Redshift estimates}

We considered all clusters with $n_{\text {gal,MC }}>1$ in the redshift estimates. Here we did not consider $n_{\text {gal,MC }}=1$ clusters because the BCG is always taken according to the selection method (Sect. 4.3.4), which leads to zero dispersion based on $500 \mathrm{MC}$ realizations. The cluster redshifts are well recovered to a level of a few per cent in all cases. There is no obvious systematic dependence on the cluster redshift. The left panels of Figs. 4-6 and Fig. 8 show the bias of the redshifts obtained in the resampled procedure from the input cluster redshifts and its dispersion based on the $500 \mathrm{MC}$ realizations. We note that the catalog of the galaxy redshifts of the HIFLUGCS is a rather clean member galaxy input catalog, probably almost free of interlopers. Uncertainties derived from the scatter in the down-sampling therefore do not account for the effect of interlopers. This artificially restricts the uncertainties of the redshift estimates to less than 0.01 . In Figs. 5 and 8 the scatter increases slightly with the number of the input galaxies, which may indicate that the scatter of redshift with a fixed number of spectroscopic members depends on the richness. Richness-dependent values of minimum numbers of galaxy redshifts for different richness populations may thus be required for a stacking analysis to ensure an equivalent scatter introduced by individual systems in the redshift estimates.

\subsubsection{Dynamical mass estimates}

There is no redshift or mass dependence in the systematic uncertainties of the dynamical mass estimates. The right panels of
Figs. 4-6 show the bias of the dynamical mass estimates obtained in the resampled procedure from the cluster total mass and its dispersion based on the $500 \mathrm{MC}$ realizations. Here we did not consider $n_{\mathrm{gal}, i}<10$ realizations, as noted in Sect. 4.4. Therefore, toward the low- $n_{\text {gal,MC }}$ end, the bias and dispersion is slightly underestimated because not all $500 \mathrm{MC}$ realizations can be used.

The dynamical mass is well recovered with a bias lower than $\sim 20 \%$ on average. The dispersion in Figs. 4-6 demonstrates the precision that the corresponding setup can reach as a function of redshift. This information can be used for the sample selection corresponding to the required precision for the purpose of mass calibration in upcoming surveys. Given the same required accuracy, the 4MOST survey can provide mass calibration up to a higher redshift than the SPIDERS survey. With ten redshifts per cluster, the dynamical mass measurement can easily be biased by a factor of two for individual clusters, which would not be suitable for a reliable mass calibration unless the clusteres were stacked. Similar to that shown in the redshift estimates, the scatter again slightly increases with the number of the input galaxies, this may indicate a dependence on richness. Richness-dependent cuts of minimum numbers of galaxy redshifts in different richness bins are also be required for a stacking analysis to ensure an equivalent scatter introduced by individual systems in the dynamical mass estimates.

\subsubsection{Limitations}

The current paper describes an ideal situation of using an input member catalog almost free of contamination from interlopers for the spectroscopic follow-up; this does not represent the reality in general. According to real surveys, we need to construct our input galaxy candidate sample for the spectroscopic followup, which cannot avoid contamination from interlopers, for example. Additionally, we need to select a number of certain member galaxies from a large catalog for the follow-up to determine the redshift and velocity dispersion, which requires knowledge of the selection effects for recovering the mass estimates in a reliable way. The input member galaxy sample for the simulation in the current paper, however, probably contains no interlopers. Neither can we quantify the selection effect because the sample is a collection of cluster galaxies from the available literature and not a spectroscopic follow-up of a well-defined photometric galaxy member sample, for instance. A further study based on mock data in simulations with known selection functions as well 

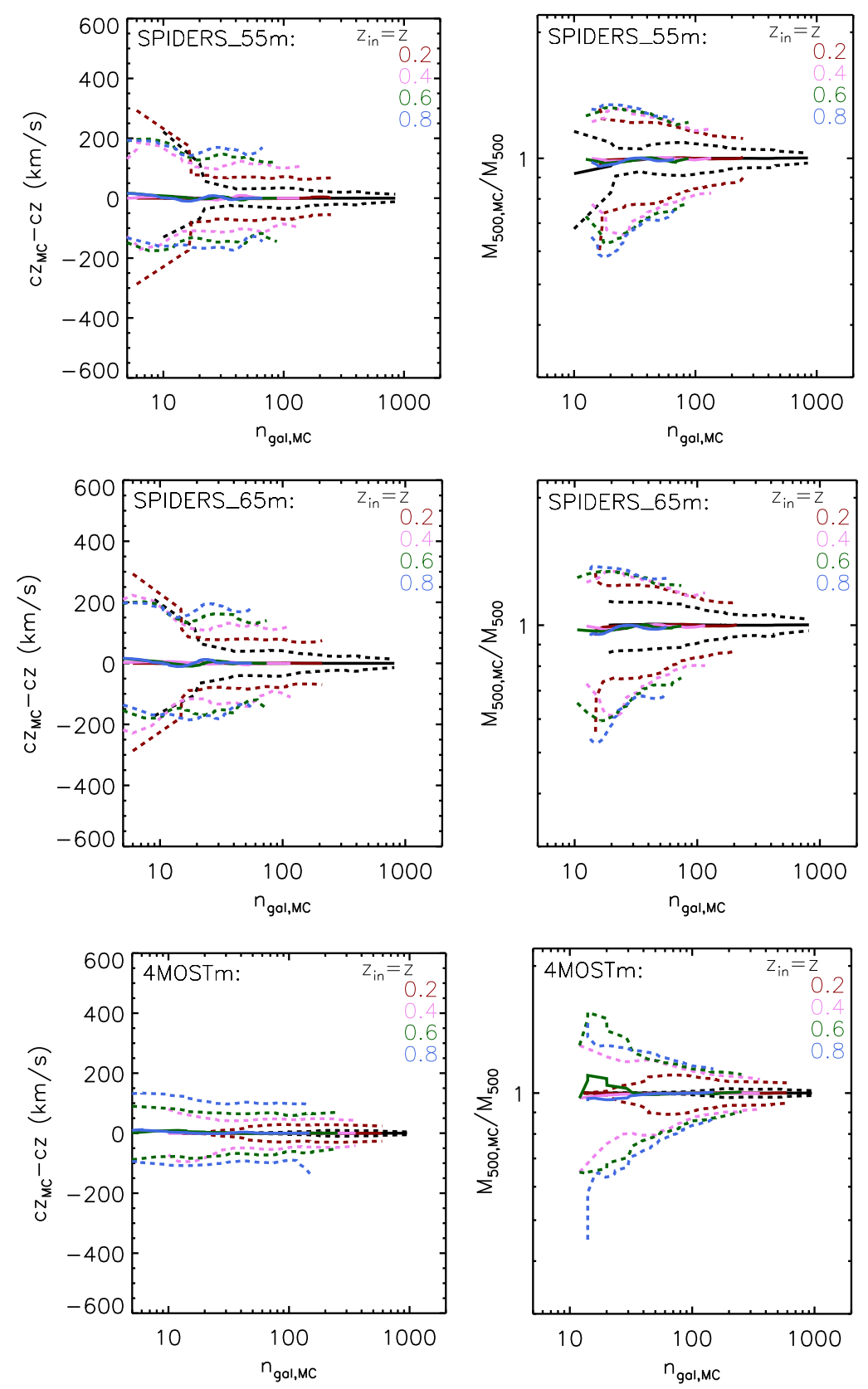

Fig. 6. Bias (solid curves) and dispersion (dashed curves) of the redshift (left panels) and dynamical mass (right panels) measurements in the SPIDERS_55m (upper panels), SPIDERS_65m (middle panels) and 4MOSTm (lower panels) setups from the input cluster values as a function of the re-sampled number of redshifts in use. The curves are smoothed with a boxcar average of the specified width of 11 to avoid spikes due to under-sampling for a few clusters.

as sufficient fore- and background galaxies in the galaxy sample will help to address these questions. Another limitation is that clusters may evolve from $z=0.8$ to the present epoch, an effect that is not fully accounted for by simply shifting the HIFLUGCS clusters to higher redshifts. It is therefore not obvious that the HIFLUGCS sample is the best test-case for forecasting the 4MOST and SPIDERS surveys at higher redshifts.

\section{Conclusions}

We analyzed $\sim 1.3 \mathrm{Ms}$ of clean XMM-Newton data and ROSAT pointed observations as well as optical spectroscopic redshifts of 13650 cluster member galaxies for all 64 HIFLUGCS clusters. Excluding 2A 0335+096 with only three redshifts, we presented the $L^{\text {cor }}-M$ relation for 63 nearby clusters of galaxies in the HIFLUGCS. For the optimal use of the optical spectroscopic surveys for high-redshift galaxy clusters and groups observed in upcoming X-ray surveys for mass calibrations, we carried out MC resampling of the galaxy members with spectroscopic redshifts and calibrated the systematic uncertainties in the redshift and dynamical mass estimates. We predicted the redshift and dynamical mass estimates assuming the SPIDERS and 4MOST optical spectroscopic survey setups, respectively, using MC resampling of the 63 HIFLUGCS clusters by placing the sample at the actual cluster redshifts as well as at redshifts of 0.2, 0.4, 0.6, and 0.8. Aiming for high-redshift cluster or group systems, we also predicted the redshift estimates based on five and ten spectroscopic members per cluster, respectively, and the mass estimates based on ten 


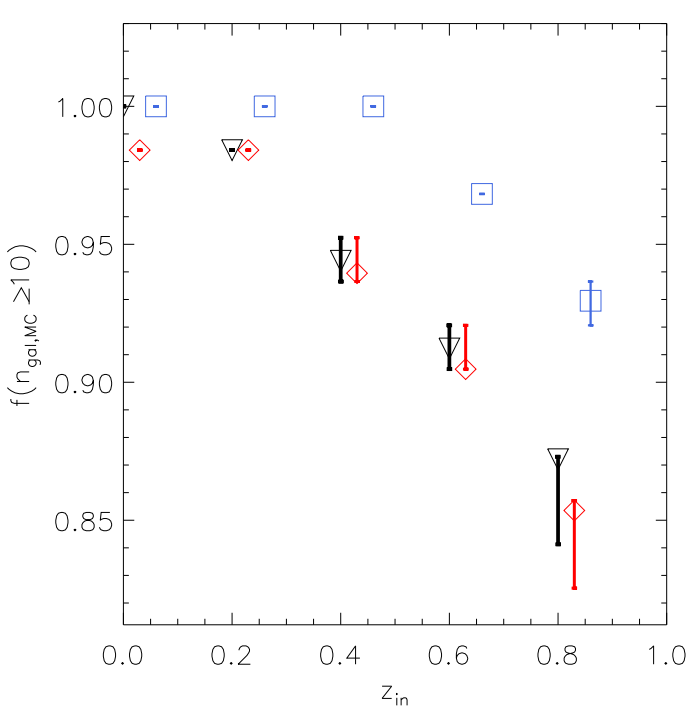

Fig. 7. Fraction of the clusters with at least ten redshifts per cluster after the resampling using the SPIDERS_55m (black triangles), SPIDERS_65m (red diamonds, with 0.03 offset) and 4MOSTm (blue squares, with 0.06 offset) setups as a function of the assigned input cluster redshift $\left(z_{\text {in }}\right)$ in the re-sampling. $z_{\text {in }}=0$ case refers to the resampled clusters at the cluster redshifts $z$.

spectroscopic members per cluster. We list the conclusions in detail as follows.

- Given sufficient numbers (i.e., $\geq 45$ ) of member galaxies in computing the dynamical masses, the $L^{\mathrm{cor}}-M$ relations agree between the disturbed and undisturbed clusters.

- The CC clusters still dominate the scatter in the $L^{\text {cor }}-M$ relation even when the core-corrected X-ray luminosity is used. This indicates that the scatter of the $L^{\text {cor }}-M$ scaling relation mainly reflects the structure formation history of the clusters.

- The dynamical masses can be measured within $10 \%$ uncertainty using the SPIDERS_55, SPIDERS_65 and 4MOST setups, which are independent of cluster redshift and mass. With ten or more redshifts per cluster, the dynamical masses can be recovered with a bias lower than $20 \%$ on average, in which the dynamical masses are underestimated for most systems.

- The bias of the cluster dynamical mass estimates increases toward the high-redshift end. The underestimation of the cluster masses on average using the SPIDERS_65m setup, in which both the collisional distance of $65^{\prime \prime}$ and magnitude cut are considered, is better than $19 \%, 28 \%, 34 \%$, and $37 \%$ in the redshift bins of $0.2,0.4,0.6$, and 0.8 . The dynamical mass is recovered with an underestimation lower than $20 \%$ up to redshift 0.6 using the 4MOSTm setup, in which both the collisional distance and magnitude cut are considered. In the redshift bin of 0.8 , the underestimation of the dynamical masses on average, according to the 4MOSTm setup, is still lower than 24\%. Assuming the SPIDERS_55m (4MOSTm) setup, the dynamical masses can be used as an independent reference blind to the X-ray observables to calibrate the cluster mass with an underestimation lower than $20 \%$ up to redshift $0.2(0.6)$ with $2 \%(3 \%)$ catastrophic outliers (i.e., fewer than ten members per cluster) in upcoming $\mathrm{X}$-ray surveys. Empirically, this bias can be corrected using a complete sample from observations or a mock sample in simulations, which includes the causes of the bias, such as contamination from fore- and background galaxies.

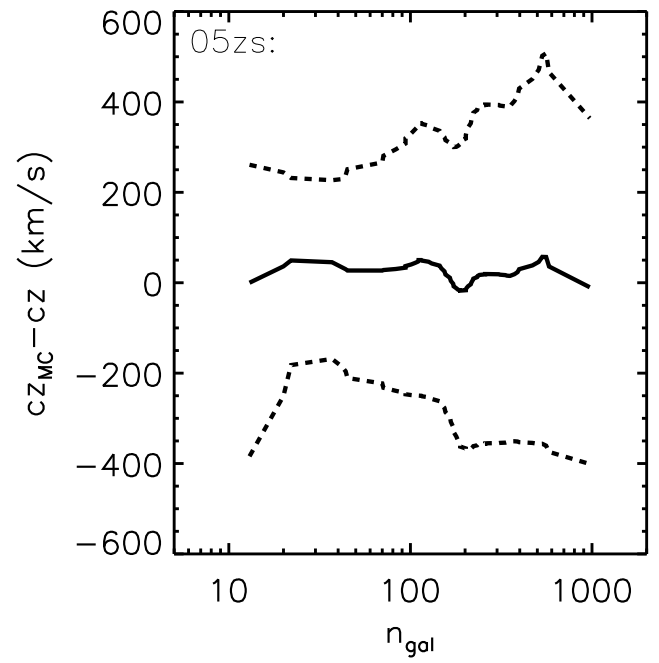

Fig. 8. Bias (solid curves) and dispersion (dashed curves) of the redshift measurements in the 05zs setup from the input cluster values versus input number of cluster galaxies. The curves are smoothed with a boxcar average of the specified width of 11 to avoid spikes due to undersampling for a few clusters. $n_{\text {gal }}$ is the input number of galaxies, and the output number of galaxies is always 5 .

Acknowledgements. The XMM-Newton project is an ESA Science Mission with instruments and contributions directly funded by ESA Member States and the USA (NASA). The XMM-Newton project is supported by the Bundesministerium für Wirtschaft und Technologie/Deutsches Zentrum für Luftund Raumfahrt (BMWi/DLR, FKZ 50 OX 0001) and the Max-Planck Society. This research has made use of the NASA/IPAC Extragalactic Database (NED) which is operated by the Jet Propulsion Laboratory, California Institute of Technology, under contract with the National Aeronautics and Space Administration. We thank Tom Dwelly for helpful discussion and an anonymous referee for the insight and expertise that improved the work. Y.Y.Z. acknowledges support by the German BMWi through the Verbundforschung under grant 50 OR 1506 T.H.R. acknowledges support from the DFG through the Heisenberg research grant RE 1462/5 and grant RE 1462/6.

\section{References}

Akritas, M. G., \& Bershady, M. A. 1996, ApJ, 470, 706

Andernach, H., Tago, E., Einasto, M., Einasto, J., \& Jaaniste, J. 2005, Nearby

Large-Scale Structures and the Zone of Avoidance, eds. A. P. Fairall, \& P. Woudt, ASP Conf. Ser., 329, 283

Andreon, S., Serra, A. L., Moretti, A., \& Trinchieri, G. 2016, A\&A, 585, A147

Applegate, D. E., von der Linden, A., Kelly, P. L., et al. 2014, MNRAS, 439, 48

Arnaud, M., Pointecouteau, E., \& Pratt, G. W. 2005, A\&A, 441, 893

Beers, T. C., Flynn, K., \& Gebhardt, K. 1990, AJ, 100, 32

Bennett, C. L., Larson, D., Weiland, J. L., et al. 2013, ApJS, 208, 20

Bernardi, M., Hyde, J. B., Sheth, R. K., Miller, C. J., \& Nichol, R. C. 2007, AJ, 133,1741

Biviano, A., Murante, G., Borgani, S., et al. 2006, A\&A, 456, 23

Blanton, M. R., \& Roweis, S. 2007, AJ, 133, 734

Bocquet, S., Saro, A., Mohr, J. J., et al. 2015, ApJ, 799, 214

Böhringer, H., Schuecker, P., Guzzo, L., et al. 2004, A\&A, 425, 367

Chen, Y., Reiprich, T. H., Böhringer, H., Ikebe, Y., \& Zhang, Y.-Y. 2007, A\&A, 466, 805

Clerc, N., Sadibekova, T., Pierre, M., et al. 2012, MNRAS, 423, 3561

Clerc, N., Merloni, A., Zhang, Y.-Y., et al. 2016, MNRAS, 463, 4490

Dawson, K. S., Schlegel, D. J., Ahn, C. P., et al. 2013, AJ, 145, 10

de Jong, R. S., Bellido-Tirado, O., Chiappini, C., et al., 2012, Proc. SPIE, 8446 $84460 \mathrm{~T}$

Diaferio, A. 1999, MNRAS, 309, 610

Ebeling, H., Edge, A.C., Allen, S. W., et al. 2000, MNRAS, 365, 1021

Eckert, D., Vazza, F., Ettori, S., et al. 2012, A\&A, 541, A57

Evrard, A. E., Bialek, J., Busha, M., et al. 2008, ApJ, 672, 122

Giacconi, R., Borgani, S., Rosati, P., et al. 2009, Astro2010: The Astronomy and

Astrophysics Decadal Survey. Science white Papers, 90

Gifford, D., \& Miller, C. J. 2013, ApJ, 768, L32

Hansen, S. M., McKay, T. A., Wechsler, R. H., et al. 2005, ApJ, 633, 122 
Hansen, S. M., Sheldon, E. S., Wechsler, R. H., \& Koester, B. P. 2009, ApJ, 699, 1333

Heymans, C., Grocutt, E., Heavens, A., et al. 2013, MNRAS, 432, 2433

Hilton, M., Romer, A. K., Kay, S. T., et al. 2012, MNRAS, 424, 2086

Hinshaw, G., Larson, D., Komatsu, E., et al. 2013, ApJS, 208, 19

Huchra, J. P., Macri, L. M., Masters, K. L., et al. 2012, ApJS, 199, 26

Hudson, D. S., Mittal, R., Reiprich, T. H., et al. 2010, A\&A, 513, A37

Hyde, J. B., \& Bernardi, M. 2009, MNRAS, 394, 1978

Israel, H., Reiprich, T. H., Erben, T., et al. 2014, A\&A, 564, A129

Israel, H., Schellenberger, G., Nevalainen, J., Massey, R., \& Reiprich, T. H. 2015, MNRAS, 448, 814

Johnston, D. E., Sheldon, E. S., Wechsler, R. H., et al. 2007, ArXiv e-prints [arXiv:0709.1159]

Kaiser, N. 1987, MNRAS, 227, 1

Katayama, H., Hayashida, K., Takahara, F., \& Fujita, Y. 2003, ApJ, 585, 687

Katgert, P., Biviano, A., \& Mazure, A. 2004, ApJ, 600, 657

Kellogg, E., Falco, E., Forman, W., Jones, C., \& Slane, P. 1990, in Gravitational Lensing, ASP Conf. Ser., 360, 141

Laureijs, R., Amiaux, J., Arduini, S., et al. 2011, ArXiv e-prints [arXiv: 1110.3193$]$

Leauthaud, A., Finoguenov, A., Kneib, J.-P., et al. 2010, ApJ, 709, 97

Malmquist, K. G. 1922, Meddelanden fran Lunds Astronomika Observatorium, Ser. I, No. 100,

Mantz, A., Allen, S. W., Rapetti, D., \& Ebeling, H. 2010, MNRAS, 406, 1759

Mantz, A. B., von der Linden, A., Allen, S. W., et al. 2015, MNRAS, 446, 2205

Maraston, C., Strömbäck, G., Thomas, D., Wake, D. A., \& Nichol, R. C. 2009, MNRAS, 394, L107

Marian, L., Hilbert, S., Smith, R. E., Schneider, P., \& Desjacques, V. 2011, ApJ, 728, L13

Maughan, B. J. 2007, ApJ, 668, 772

Merloni, A., Predehl, P., Becker, W., et al. 2012, ArXiv e-prints [arXiv: 1209. 3114]

Munari, E., Biviano, A., Borgani, S., Murante, G., \& Fabjan, D. 2013, MNRAS, 430, 2638

Nandra, K., Barret, D., Barcons, X., et al. 2013, ArXiv e-prints [arXiv: 1306.2307]

Navarro, J. F., Frenk, C. S., \& White, S. D. M. 1997, ApJ, 490, 493

Old, L., Gray, M. E., \& Pearce, F. R. 2013, MNRAS, 434, 2606

Owers, M. S., Nulsen, P. E. J., \& Couch, W. J. 2011, ApJ, 741, 122

Pacaud, F., Pierre, M., Adami, C., et al. 2007, MNRAS, 382, 1289

Peng, C. Y., Ho, L. C., Impey, C. D., \& Rix, H.-W. 2002, AJ, 124, 266

Pillepich, A., Porciani, C., \& Reiprich, T. H. 2012, MNRAS, 422, 44

Planck Collaboration XVI. 2016, 594, A16

Planck Collaboration XIII. 2016, 594, A13

Pointecouteau, E., Reiprich, T. H., Adami, C., et al. 2013, ArXiv e-prints [arXiv: 1306.2319]
Poole, G. B., Fardal, M. A., Babul, A., et al. 2006, MNRAS, 373, 881

Popesso, P., Böhringer, H., Romaniello, M., \& Voges, W. 2005, A\&A, 433, 415

Pratt, G. W., Croston, J. H., Arnaud, M., \& Böhringer, H., 2009, A\&A, 498, 361 Predehl, P., Böhringer, H., Brunner, H., et al. 2010, Proc. SPIE, 7732

Rabitz, A., Zhang, Y.-Y., Schwope, A., et al. 2017, A\&A, 597, A24

Reiprich, T. H., \& Böhringer, H. 2002, ApJ, 567, 716

Reichert, A., Böhringer, H., Fassbender, R., \& Mühlegger, M. 2011, A\&A, 535, A4

Reyes, R., Mandelbaum, R., Hirata, C., Bahcall, N., \& Seljak, U. 2008, MNRAS, 390, 1157

Riess, A. G., Macri, L., Casertano, S., et al. 2011, ApJ, 730, 119

Rines, K., \& Diaferio, A. 2006, ApJ, 132, 1275

Rines, K. J., Geller, M. J., Diaferio, A., \& Hwang, H. S. 2016, ApJ, 819, 63

Roettiger, K., Loken, C., \& Burns, J. O. 1997, ApJS, 109, 307

Santos, J. S., Rosati, P., Tozzi, P., et al. 2008, A\&A, 483, 35

Saro, A., Mohr, J. J., Bazin, G., \& Dolag, K. 2013, ApJ, 772, 47 Sereno, M. 2015, MNRAS, 450, 3665

Sereno, M., \& Ettori, S. 2015, MNRAS, 450, 3675

Schlegel, D. J., Finkbeiner, D. P., \& Davis, M. 1998, ApJ, 500, 525

Schlegel, D., Abdalla, F., Abraham, T., et al. 2011, ArXiv e-prints [arXiv: 1106.1706]

Schrabback, T., Hartlap, J., Joachimi, B., et al. 2010, A\&A, 516, A63

Serra, A. L., \& Diaferio, A. 2013, ApJ, 768, 116

Stanek, R., Evrard, A. E., Böhringer, H., Schuecker, P., \& Nord, B. 2006, ApJ, 648, 956

Stanek, R., Rasia, E., Evrard, A. E., Pearce, F., \& Gazzola, L. 2010, ApJ, 715, 1508

Takey, A., Schwope, A., \& Lamer, G. 2013, A\&A, 558, A75

Tyler, K. D., Rieke, G. H., \& Bai, L. 2013, ApJ, 773, 86

Tyler, K. D., Bai, L., \& Rieke, G. H. 2014, ApJ, 794, 31

Vikhlinin, A., Burenin, R. A., Ebeling, H., et al. 2009a, ApJ, 692, 1033

Vikhlinin, A., Kravtsov, A. V., Burenin, R. A., et al. 2009b, ApJ, 692, 1060

von der Linden, A., Mantz, A., Allen, S. W., et al. 2014, MNRAS, 443, 1973

Weiner, B. J., Willmer, C. N. A., Faber, S. M., et al. 2006, ApJ, 653, 1049

Wu, H.-Y., \& Huterer, D. 2013, MNRAS, 434, 2556

Wu, X.-P., Fang, L.-Z., \& Xu, W. 1998, A\&A, 338, 813

Wu, X.-P., Xue, Y.-J., \& Fang, L.-Z. 1999, ApJ, 524, 22

Zhang, Y.-Y., \& Wu, X.-P., 2003, ApJ, 583, 529

Zhang, Y.-Y., Finoguenov, A., Böhringer, H., et al. 2007, A\&A, 467, 437

Zhang, Y.-Y., Finoguenov, A., Böhringer, H., et al. 2008, A\&A, 482, 451

Zhang, Y.-Y., Reiprich, T. H., Finoguenov, A., Hudson, D. S., \& Sarazin, C. L. 2009, ApJ, 699, 1178

Zhang, Y.-Y., Okabe, N., Finoguenov, A., et al. 2010, ApJ, 711, 1033

Zhang, Y.-Y., Andernach, H., Caretta, C. A., et al. 2011, A\&A, 526, A105

Zhang, Y.-Y., Verdugo, M., Klein, M., \& Schneider, P. 2012, A\&A, 542, A106 


\section{Appendix A: Effect of flux loss of the aperture magnitude}

\section{A.1. Fraction of recovered flux}

We used a Sérsic profile for the two-dimensional (2D) surface brightness profiles of galaxies (e.g., Peng et al. 2002), $\Sigma(x, y) \propto$ $\exp \left[-\kappa\left(\frac{r_{\mathrm{ell}}}{R_{\mathrm{e}}}\right)^{1 / n}-1\right]$ with $r_{\mathrm{ell}}=\left[|x|^{(c+2)}+\left|\frac{y}{q}\right|^{(c+2)}\right]^{1 /(c+2)}$. The parameter $q$ is the ellipticity, and we used its distribution observed in SDSS (Fig. 3 in Hyde \& Bernardi 2009, peaked at $\sim 0.8$ ). For a de Vaucouleurs profile, $c=0, n=4, \kappa=7.67$ for $n=4$, and $\Sigma(x, y) \propto \exp \left[-7.67\left(r_{\mathrm{ell}} / R_{\mathrm{e}}\right)^{1 / 4}\right]$. Following Bernardi et al. (2007), we computed the effective radius in units of $\mathrm{kpc}$ from the $r$-band absolute magnitude for a passive, elliptical galaxy, $\left\langle\log R_{\mathrm{e}} \mid M_{r}\right\rangle=-0.681\left(M_{r}+21\right) / 2.5+0.343$, using the spectral energy distribution template in Maraston et al. (2009).

We assumed 1". 4 seeing for SPIDERS and $1^{\prime \prime}$ seeing for 4MOST (private communication with T. Dwelly), in which the seeing is better than the given value for $90 \%$ of the time. The diameter of the aperture is $\varnothing=2^{\prime \prime}$ for SPIDERS and 1".5 for 4MOST. The resulting flux was computed by integrating the surface brightness profile convolved with the seeing within the aperture. The fractions of recovered flux $f_{\text {synth }}$ in the fibers of the SPIDERS and 4MOST surveys are shown in Fig. A.1. Our results are consistent with that of the objects classified as "LRG" or
"GALAXY", which have spectroscopic redshifts from the BOSS in SDSS DR11.

\section{A.2. Effect on our sampling}

For galaxies with an intrinsic magnitude only slightly brighter than the limiting magnitude cut, its aperture magnitude may become fainter than the limiting magnitude when considering flux loss. We inspected this effect on our sampling by comparing the aperture magnitudes of the member galaxies used in the resampling with the limiting magnitude cut. Although there is quite a dramatic difference $(\sim 1-1.5 \mathrm{mag})$ between the total magnitude and the $2^{\prime \prime}$ aperture magnitude, we found that the aperture magnitudes of all member galaxies are well above the limiting magnitude cut for the clusters at $z_{\text {in }}<0.4$ and $z_{\text {in }}=0.8$. This is because the galaxies are much brighter than the limiting magnitude at the low redshift of $z_{\text {in }}=0.2$, and the angular sizes of the galaxies are well within the aperture size at the high redshift of $z_{\text {in }}=0.8$ such that flux loss plays no role. At redshifts of $z_{\text {in }}=0.4$ and 0.6 , the aperture magnitude considering flux loss leads to a number of member galaxies fainter than the limiting magnitude in a couple of clusters among 63 clusters in the resampling. No more than three out of 63 clusters are affected by the flux loss. We therefore consider this effect negligible. Flux loss plays a main role in degrading the spectral quality, which is not part of this study.
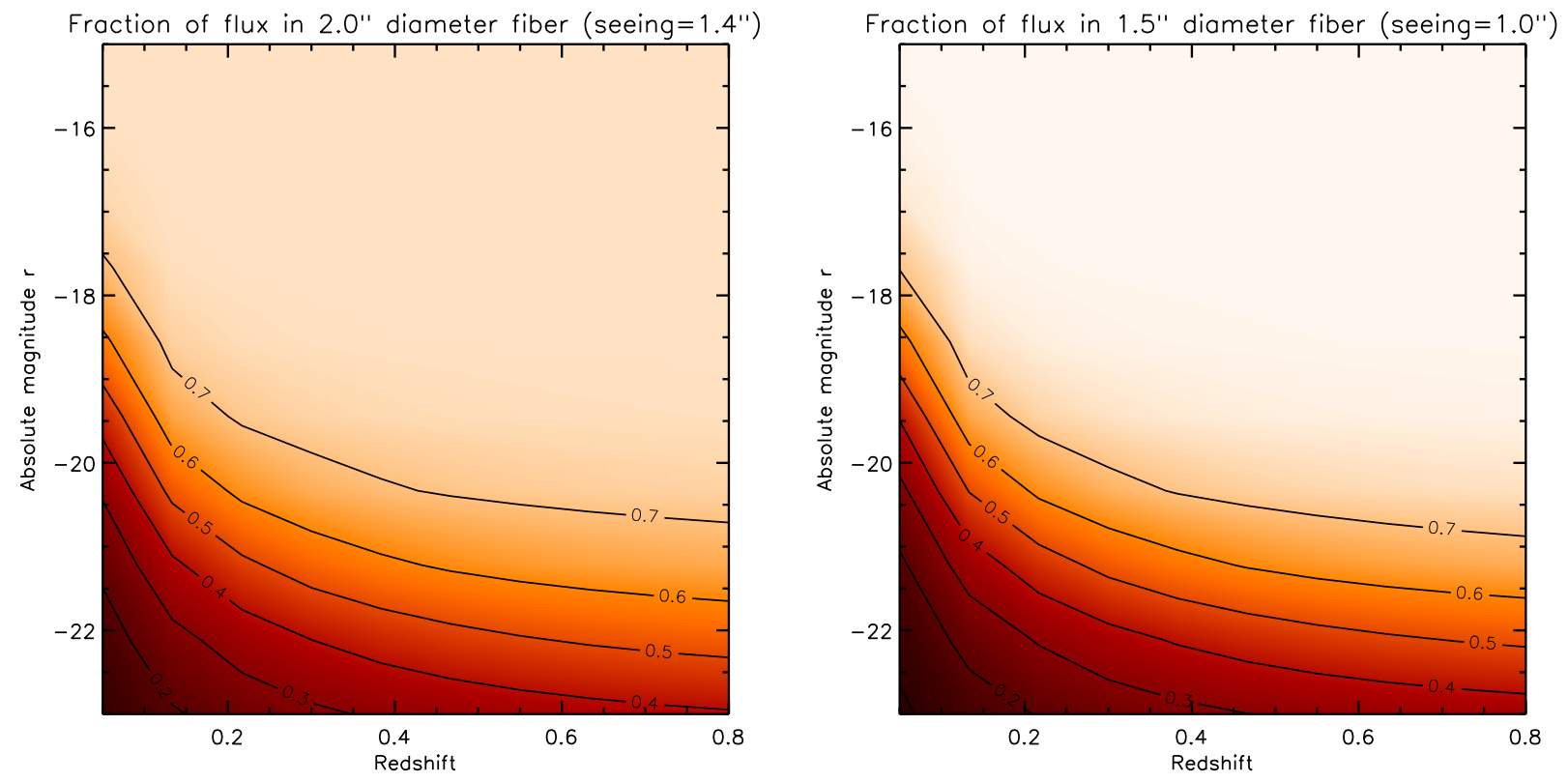

Fig. A.1. Fractions of recovered flux $f_{\text {synth }}$ in the fibers of the SPIDERS (left panel) and 4MOST (right panel) configurations. The $x$-axis and $y$-axis are the galaxy redshift and $r$-band absolute magnitude, respectively. 